\title{
A survey on dynamic spectrum access protocols for distributed cognitive wireless networks
}

\author{
Pinyi Ren", Yichen Wang, Qinghe Du and Jing Xu
}

\begin{abstract}
With the rapid development of wireless communications technologies, radio spectrum has become a type of extremely scarce resources in meeting the increasing demands for broadband wireless services. However, the traditional static spectrum allocation policy leads to severe spectrum underutilization and spectrum shortage problems. The cognitive radio (CR) technology can detect the occupancy of the spectrum and enable the dynamic spectrum access (DSA) to fill the spectrum hole caused by the static allocation policy, and thus has been widely recognized as an efficient approach to solve the above problems. The distributed cognitive wireless network (CWN), which does not have central entities, is one of the major networking architectures applying the CR technology. Correspondingly, the design of DSA in distributed CWNs is crucial, yet challenging, to increasing the utilization efficiency of the wireless spectrum with dynamically-varying occupancy statuses. In this article, we present a survey on DSA protocols for distributed CWNs. In particular, we first address the challenges in the design and implementation of distributed DSA protocols. Then, we categorize the existing distributed DSA protocols based on different criteria, such as spectrum sharing modes, spectrum allocation behaviors, spectrum access modes, the usage of common control channel, spectrum usage strategies, the number of radios, and spectrum sensing techniques. We also discuss the advantages and disadvantages of each category under diverse classification criterion. Moreover, we make a comprehensive survey of the state-of-the-art distributed DSA protocols using different spectrum access modes, which can be categorized into contention-based, time-slotted, and hybrid protocols. Through the study, we find out that most of distributed DSA protocols fall into the contention-based and hybrid protocols. In addition, the ongoing standardization efforts are also reviewed. Finally, several open research issues for the distributed DSA protocols are presented, such as spectrum handoff based protocols, spectrum prediction based protocols, adaptation of the spectrum-sharing modes, protocols with cooperative spectrum sensing, as well as distributed collision avoidance mechanisms.
\end{abstract}

\section{Introduction}

With the rapid development of the wireless technologies, the demand for the spectrum resources is constantly increasing. However, current spectrum utilization efficiency is very low because of the traditional static spectrum allocation policy, where a particular portion of spectrum can be only used by a specific type of wireless communications systems. While some unlicensed spectrum, such as ISM (industrial, scientific and medical) frequency bands, can be freely used by any users, they have been too crowded to accommodate more wireless applications. In addition, the utilization statuses of the licensed spectrum change significantly over time, space,

\footnotetext{
* Correspondence: pyren@mail.xjtu.edu.cn

School of Electronic and Information Engineering, Xi'an Jiaotong University, Shaanxi Province, Xi'an 710049, China
}

\section{Springer

() 2012 Ren et al; licensee Springer. This is an Open Access article distributed under the terms of the Creative Commons Attribution License (http://creativecommons.org/licenses/by/2.0), which permits unrestricted use, distribution, and reproduction in any medium, provided the original work is properly cited. and frequency domain. Therefore, allowing unlicensed users to dynamically access the licensed spectrum which are underutilization, such as UHF/VHF TV bands, is an effective way to improve the spectrum utilization efficiency [1-5].

Cognitive radio (CR) technology, which was first proposed by Mitola [6], is a promising approach to realize dynamic spectrum access (DSA) to overcome the above problems. DSA can be divided into two categories, which are spectrum overlay and spectrum underlay, respectively. In the spectrum overlay, the unlicensed users, also known as secondary users (SUs), can only use the spectrum resources that are not currently used by licensed users, also known as primary users (PUs). In the spectrum underlay, the SUs subject to certain 
interference constraints, the SUs are allowed to use the licensed spectrum even when the PUs are active.

In traditional wireless networks, efficient spectrum allocation and access scheduling can significantly improve the network performance. Thus, designing spectrum access protocols ${ }^{\mathrm{a}}$ is one of the most important targets for wireless networks [7-9]. Unlike the static spectrum allocation in traditional multi-channel wireless networks, the available spectrum resources in cognitive wireless networks (CWNs) varies with time, frequency, and space. Consequently, the existing multi-channel spectrum access protocols cannot be directly applied into CWNs. In distributed CWNs, the design of distributed DSA protocols is an even more challenging task due to the absence of the central control entities, such as the base stations and access points. During the past ten years, DSA protocols for CWNs had attracted a great deal of research attention [10-16]. As discussed in these existing studies, an efficient DSA protocol for CWNs should support the following four functions:

- Transparency for PUs: The essential of CR is that SUs should dynamically find, track, and choose available spectrum resources, which varies with time, frequency, and space, only based on SUs' local observation and negotiation among SUs. Therefore, no interaction or coordination between SUs and PUs should exist, i.e., SUs should be transparent to PUs.

- Collision avoidance: In distributed CWNs, the collision avoidance suggests two aspects. On the one hand, the DSA protocols need to avoid the collisions among different SUs through coordinating their spectrum access. On the other hand, the collisions between SUs and PUs also need to be avoided, which can be implemented by applying accurate spectrum sensing or imposing the stringent interference power constraints.

- Accurate spectrum sensing: The accuracy of spectrum sensing will significantly affect the performance of CWNs. However, the local spectrum sensing is often unreliable due to the impact of path loss, channel fading, shadowing, noise uncertainty, etc. Therefore, some novel mechanisms, such as cooperative spectrum sensing, are desirable. However, implementing such mechanisms in distributed CWNs is still a challenging task.

- Efficient dynamic spectrum allocation: In CWNs, if spectrum resources are efficiently allocated, not only the average network throughput can be increased and collisions among different SUs can be reduced, but also the fairness can be improved. Thus, DSA protocols should provide efficient dynamic spectrum allocation, which however is still hard to achieve in distributed CWNs because of the completely distributed networking architecture.

In addition, the DSA protocol is a bridge connecting the physical layer and upper protocol layers. Specifically, it cannot only exploit available spectrum resources based on spectrum sensing implemented in the physical layer, but also can help the network layer establish the route by providing the corresponding available spectrum information and satisfy the quality-of-service (QoS) requirement of the application layer by using the efficient dynamic resource allocation.

The rest of this article is organized as follows. Section 2 presents the challenges of distributed DSA protocols. In Section 3, the existing distributed DSA protocols are classified based on different criterion. Then, we review the existing distributed DSA protocols in Section 4. The ongoing standardization efforts are reviewed in Section 5. Several open research issues are addressed in Section 6. The article concludes with Section 7.

\section{Challenges for distributed dynamic spectrum access protocols}

Cognitive wireless networks (CWNs) aim to 1) utilize the $\mathrm{CR}$ technology to provide available spectrum opportunities for cognitive users, 2) reduce the burden for crowded unlicensed channels while large portions of the licensed spectrum bands remain vacant at the same time and location, and 3) improve the overall spectrum utilization efficiency. In the well-known IEEE 802.22 standard, which is the first CR standard, the CWNs can provide throughput of $1.5 \mathrm{Mb} / \mathrm{s}$ in the downstream [1,2]. Generally speaking, due to the absence of central controllers, the throughput of distributed networks is worse than that of centralized networks. However, lots of existing distributed DSA protocols are designed based on the IEEE 802.11 DCF standard (to be discussed in Section 4). As shown in [17], the maximal throughput of each channel for the IEEE $802.11 \mathrm{DCF}$ standard is only about $0.82 \mathrm{Mb} / \mathrm{s}$ when available bandwidth is $1 \mathrm{Mbps}$. If we take the multihop transmission, mobility, and time-varying channel availability into consideration, the maximal throughput of distributed CWNs will be even lower than that of IEEE 802.11 DCF standard, which is caused by the fact that the available spectrum bands are not sufficiently utilized. In order to improve the network performances of distributed CWNs, several challenges should be considered, such as the common control channel (CCC) problem, spectrum sensing scheduling strategy, power control mechanism, multichannel hidden, and exposed terminal problems, access coordination mechanism, and mobility. 


\subsection{Common control channel problem}

Using CCC is an effective method to guarantee the reliable control information exchanges for wireless communications systems. In traditional distributed multichannel wireless networks, the CCC can be one of the licensed channels [18]. In centralized CWNs, the central controller (e.g., base station or access point) can assign a certain dedicated channel as CCC, and thus all control information can be reliably exchanged between the central controller and SUs. However, compared with the traditional distributed multi-channel wireless networks and centralized CWNs, determining which channel is used as the CCC in distributed CWNs is more difficult due to the time-varying spectrum resources and the absence of central controller. Recently, employing a dedicated CCC for the distributed CWN becomes a widely accepted assumption. The dedicated CCC can be implemented by two approaches, namely, licensed CCC and unlicensed CCC [19]. In most existing studies using the dedicated CCC, a licensed channel for the distributed CWN is assumed to exist to act as the dedicated CCC [20-24]. In this way, control packets of the distributed CWN can be exchanged without interference from other systems. However, such licensed CCC may not exist in practical CWNs due to the spectrum allocation policy. Using an unlicensed CCC to act as the CCC is another method [25]. However, such commonly available channel may not be found because the available spectrum resources of different SUs may be totally different. Moreover, using the dedicated CCC will also cause the CCC saturation problem, which often degrades the network performances. In order to solve the above problems, control packets can be transmitted separately on different channels, such as the hoppingbased CCC [26,27]. In this method, the control packets can be exchanged on all unlicensed channels according to the pre-defined hopping sequence. Although the CCC saturation problem can be effectively alleviated, the hopping-based CCC requires stringent network-wide synchronization, which will significantly increase the complexity and cost.

\subsection{Spectrum sensing scheduling strategy}

Spectrum sensing, which is one of the most important functions of CWNs, can provide available spectrum resources for CWNs. Lots of spectrum sensing methods have been proposed [28-32]. Clearly, spectrum sensing will consumes a certain amount of time, which decreases data transmission time. If the spectrum-sensing time is too long, the network throughput of CWNs will be severely degraded. On the one hand, [33] shows that the optimal sensing time maximizing the network throughput while sufficiently protecting PUs exists. It is desirable to dynamically determine the sensing time to minimize the spectrum sensing overhead, such that the network performances can be improved. On the other hand, as the spectrum occupation statues of PUs are time-varying, the DSA protocols should also contain three spectrum sensing decisions, i.e., when to sense, which channel to sense, and how many channels should be sensed [10]. If these decisions can be correctly made, the spectrum sensing can be performed in the most efficient way, which can significantly improve the network performances. Based on the above discussions, the spectrum sensing scheduling strategy should be embedded into the DSA protocols and carefully designed. In centralized CWNs, the sensing scheduling strategy can be easily determined by central controllers. However, due to the absence of such central control entity, the sensing scheduling strategy are more challenging for the distributed CWNs. In distributed CWNs, the sensing scheduling strategy can only be made by each $\mathrm{SU}$ and the strategies made by different SUs are usually not the same. Generally speaking, the strategies made by different SUs will mutually affected, which causes that the spectrum sensing scheduling strategy of each SU could frequently change and the optimal sensing schedule strategy cannot be easily derived. Although some investigations have been conducted for the single point-topoint wireless link [34-39], where there is only one source-destination node pair, designing efficient spectrum sensing strategy for the network is still a challenging task.

\subsection{Power control mechanism}

Because the power control mechanism can reduce the mutual interferences among neighbor nodes, it can efficiently improve the spatial reuse efficiency and significantly increase the network throughput. Moreover, it can also help users save energy, which is very important to the system with limited energy. Therefore, the power control mechanism is a critically important component for the spectrum access protocols. Although some distributed DSA protocols with power control mechanism have been proposed, such as [40-44], etc., designing efficient power control mechanism is still a complex and challenging task for distributed CWNs. First, compared with traditional distributed wireless networks, not only the interferences among different SUs should be considered, but also the interference constraints imposed by PUs should be taken into consideration in the distributed CWNs. Second, compared with the centralized networks, the power control mechanism for distributed CWNs will be implemented through a completely distributed manner, which makes the interference coordination among multiple SUs more difficult. Moreover, as the investigation in [45], the transmit power of the SU will also impact the occurrence of the available 
spectrum opportunity. Specifically, a channel can be viewed as a opportunity to a source-destination pair if and only if the PU receivers cannot be interfered by the source and the transmission sent by the source can be successfully received by the destination. If we increase the transmit power of the source, although the signalto-interference plus noise ratio (SINR) at the destination can be improved, which leads to a higher probability that the communication can be successfully performed, more PU receivers may be interfered by the source, which causes less available spectrum opportunities. Therefore, the power control mechanism is a challenge for distributed DSA protocols.

2.4 Multi-channel hidden and exposed terminal problems As there are multiple available spectrum bands in the distributed CWNs, the multi-channel hidden terminal problem, which is a challenge for the traditional distributed multi-channel wireless networks and first proposed in [18], is also an important issue in the distributed CWNs. The multi-channel hidden terminal problem is that, a sending node may not hear the ongoing reception of its neighbor receiving node because different nodes can operate on different channels, which results that the sending node may transmit its data packet on the channel that is the same with its neighbor receiving node. Therefore, data packet collisions will be caused by the multi-channel hidden terminal problem. It is clear that the multi-channel hidden terminal problem is harmful for the network performances. The multi-channel hidden terminal problem can be solved by using a control radio to operate on the $\mathrm{CCC}[20-22,40]$, but additional costs will be caused. Moreover, the multichannel hidden terminal problem is not well solved in the distributed CWNs without using the dedicated CCC $[27,46]$.

The multi-channel exposed terminal problem is another critical issue in the distributed CWNs, but has been neither well understood nor comprehensively studied [40]. So-called multi-channel exposed terminal problem is that, when one sending node discovers its neighbor nodes are sending data packets in some data channels through carrier sense, the sending node will give up choosing those channels for transmission. Obviously, the available channels for the sending node are remarkably reduced. Therefore, the network throughput will be seriously degraded.

\subsection{Access coordination mechanism}

In the distributed wireless networks, due to the absence of the central controller, all users will compete for the spectrum access in a totally distributed manner, which causes that the collisions among competing users are inevitable, thus significantly decreasing the network performances. Therefore, the access coordination mechanism is critically important to the distributed network and should be considered by the distributed DSA protocols. In order to reduce the collisions among the competing SUs, several methods have been proposed. Because lots of distributed DSA protocols are designed based on the IEEE 802.11 DCF standard (to be discussed in Section 4), the collisions can be reduced by optimizing the parameters in this mechanism. As shown in [17], the length of the contention window and the transmission probability that a user transmits in a randomly chosen slot are two critically important parameters. For a given number of users, the optimal values of these two parameters minimizing the number of collisions and maximizing the network throughput exist. The similar method can be used in the distributed DSA protocols. The second method is to allow SUs to switch to the channel with better conditions if the current channel is too crowded [25,47]. In this way, the transmission rates of SUs can be improved and the collisions can be reduced. The third approach is to separate the spectrum access of different source-destination pairs on different channels [26,27]. Moreover, utilizing the directional antenna is another efficient approach to reduce the collisions $[42,48,49]$. This is because the spatial reuse efficiency can be significantly improved, which results that the interferences among SUs can be reduced and more SUs can access the spectrum simultaneously.

\subsection{Mobility}

Mobility is an important issue that needs to be seriously considered for the distributed DSA protocol design. First, most distributed DSA protocols rely on the time synchronization for better spectrum access coordination and some distributed DSA protocols even need the stringent network-wide synchronization, such as the time slotted protocols which will be introduced in Section 3.3. However, mobility of nodes may cause the drift of the reference clock, which will significantly degrade the network performance. Second, mobility of SUs may result tremendous changes of available spectrum sources, thus causes that the DSA protocols cannot perform efficient resource allocation and access coordination.

\section{Classifications for distributed dynamic spectrum access protocols}

During past ten years, a large number of distributed DSA protocols for CWNs are investigated. These protocols can be classified according to different criteria, such as spectrum sharing modes, spectrum allocation behaviors, spectrum access modes, the usage of common, number of radios, spectrum usage strategies, spectrum sensing techniques, etc. Figure 1 presents such classifications based on the above criteria. 


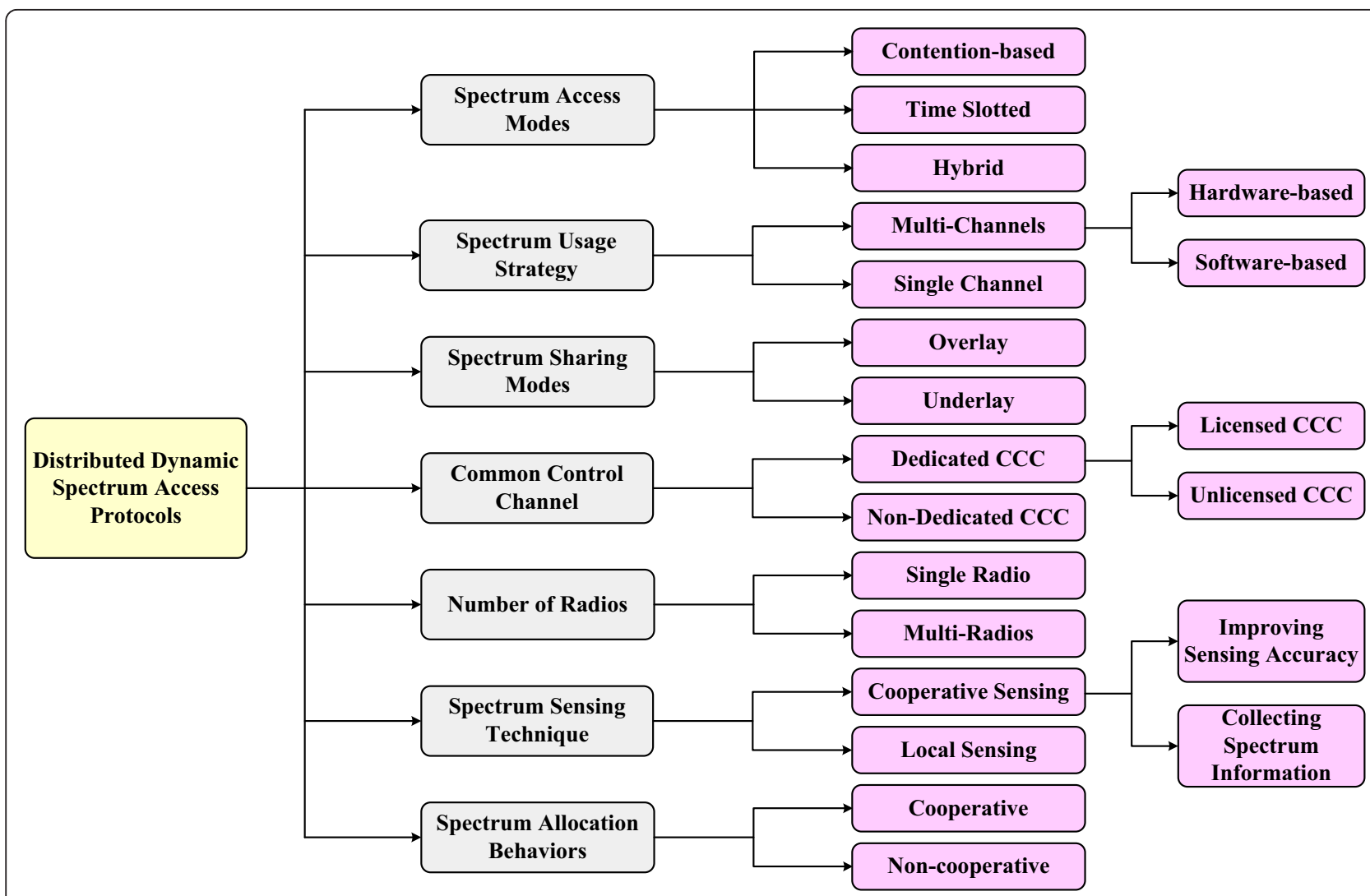

Figure 1 Classification of dynamic spectrum access protocols for cognitive wireless networks.

\subsection{Spectrum sharing mode based classification}

According to different spectrum sharing modes, distributed DSA protocols can be classified into two categories, i.e., spectrum overlay and spectrum underlay [10,15]. In overlay protocols, SUs can only access the spectrum that are not currently occupied by PUs, which causes that spectrum sensing is specially important $[21,22,24,26,35,40,50]$. In the underlay protocols, SUs are allow to share the spectrums that are occupying by PUs, only need to subject to the pre-defined maximal transmit power, i.e., power mask $[41,44]$.

The overlay protocols are more suitable for the scenario that the spectrum usage status of PUs changes slowly. In this case, SUs can sufficiently utilize their power resources on those vacant spectrum bands. However, if the spectrum usage status of PUs changes quickly, SUs may not track the variation of PUs' traffics. Moreover, SUs cannot transmit their data packets all the time because spectrum sensing will be performed periodically, which will degrade the network throughput in some degree. Compare with the overlay protocols, the underlay protocols do not perform spectrum sensing, thus are more suitable for the scenario that the spectrum usage status of PUs changes quickly. However, SUs cannot sufficiently utilize their power resources because of the interference power constraints. In addition, calculating the maximal transmit power of SUs that will not interrupt PUs' transmissions is a challenging task [43].

\subsection{Spectrum allocation behavior based classification}

According to different spectrum allocation behaviors, distributed DSA protocols can be divided into two categories, which are Cooperative and Non-cooperative, respectively [51]. The cooperative DSA protocols aim to maximize the whole network performance through the cooperation among SUs $[41,44,52]$. On the other hand, in non-cooperative protocols, SUs aim to optimize their own performance independently through their local observations and decisions [20,22,24-26,40].

Because the non-cooperative DSA protocols are only designed to maximize the performance (such as throughput, delay, etc.) of each source-destination pair, this kind of protocols have simple architectures and low computation complexity. Generally speaking, the cooperative DSA protocols try to achieve the global optimization, which causes that this kind of protocols need to solve complex optimization problems. Although the cooperative DSA protocols have more complex architectures and higher computation complexity than the non- 
cooperative protocols, the performance of the former ones usually better than that of the latter ones.

\subsection{Spectrum access mode based classification}

According to different spectrum access modes, distributed dynamic spectrum access protocols can be divided into three categories, which are Contention-based, Slotted, and hybrid protocols, respectively [11,14]. In the contention-based protocols, spectrum access is usually implemented by carrier sense multiple access with collision avoidance (CSMA/CA) mechanism, which is similar as the mechanism in IEEE 802.11 DCF standard $[20,21,23,24,41,43,44,50]$. In the slotted protocols, a unique slot is assigned for each $\mathrm{SU}$, thus interferencefree transmissions are available for SUs [25,40]. Hybrid protocols aim to derive the tradeoff between the contention-based protocols and slotted protocols. This kind of protocol is usually based on the ON/OFF model. In each slot, SUs compete for the spectrum according to the contention-based protocols [22,26,27,35,39,42,52].

Generally speaking, the contention-based protocols have the simplest architectures than the other kinds of protocols. However, the spectrum utilization efficiency and network performance are usually the lowest due to the serious packet collisions and inefficient spectrum competitions. The slotted protocols can usually achieve the best network performance compared with the contention-based and hybrid protocols. However, the protocol design of this kind of protocols is the most complex because a series of problems are needed to be considered, such as synchronization mechanism and slot allocation strategy. The hybrid protocols try to achieve the tradeoff between contention-based and slotted protocols. Therefore, the performance of hybrid protocols usually outperforms that of the contention-based protocols and the complexity is lower than that of the slotted protocols.

\subsection{Common control channel based classification}

$\mathrm{CCC}$ is another metric to classify the distributed DSA protocols. Based on whether the dedicated CCC is used, DSA protocols can be divided into two categories, i.e., dedicated CCC based and non-dedicated CCC based protocols. In the dedicated CCC based protocols, one channel is assumed to be dedicated for exchanging control packets. This kind of protocols can be further divided into two sub-categories according to whether the CCC is licensed to the CWNs, which are licensed CCC based and unlicensed CCC based protocols, respectively. Compared with the licensed CCC based protocols [20-22,41,53-55], in which the dedicated CCC are assumed to be licensed to CWNs, the unlicensed CCC based protocols will select a unlicensed channel as the dedicated CCC [25]. In the non-dedicated CCC based protocols, there is no dedicated $\mathrm{CCC}$ in the $\mathrm{CWNs}$, which results that the control packets will be exchanged over multiple unlicensed channels $[26,27,35,46,56]$.

Utilizing the dedicated CCC for control packet exchanges is a widely accepted assumption for distributed DSA protocols. In the dedicated CCC based protocols, because all control packets are exchanged on one channel, the SUs can conveniently obtain the information of their neighbor nodes through listening to the dedicated CCC, which can efficiently simplify the protocol architectures. However, some drawbacks are existed in this kind of protocols. First, a licensed channel may not exist in realistic CWNs. Second, it is easy to be affected by the PUs' traffic if an unlicensed channel acts as the dedicated CCC. Third, utilizing dedicated CCC may cause the CCC saturation problem. Although nondedicated CCC based protocols can efficiently solve above problems, the control packet exchanging mechanism should be well designed, which causes that this kind of protocols usually have more complex architecture.

\subsection{Spectrum usage strategy based classification}

Based on different spectrum usage strategies, existing distributed DSA protocols can be divided into two categories, which are single channel based and multi-channel based protocols, respectively. Compared with the single channel based protocols $[20,21,23,25,26,35,40,53,54]$, in which each source-destination pair can only use one channel for their data transmissions, the multi-channel based protocols allow the source and destination nodes to utilize multiple channels simultaneously. According to different methods that use multiple channels simultaneously, the multi-channel based protocols can be further divided into two sub-categories, i.e., hardware-based and software-based protocols. In the hardware-based protocols $[43,44]$, multiple data radios are equipped for one SU with each radio operating on one channel. On the contrary, the software-based protocols only need one data radio per SU based on the channel aggregation technique $[22,24,39,41,46,50,52,55,56]$.

Although the single channel based protocols have much simpler architectures than the multi-channel based protocols, the data transmission rate of the source-destination pair is lower than that of the latter kind of protocols. However, multi-channels based protocols usually need to design more complex mechanisms to guarantee the fairness of protocols and efficiently allocate spectrum and transmit power. Moreover, the multi-channel based protocols often have higher cost than the single-channel based protocols because more radios are needed.

\subsection{Number of radios based classification}

Based on the number of radios for each SU, the DSA protocols can be divided into single radio based and 
multi-radios based protocols. In the single radio based protocols $[22,24,25,46,50,55,56]$, only one radio is equipped for each SU. On the contrary, at least two radios are needed for each SU [20-22,26,41,43,44,52,53].

Compared with the single radio based protocols, the multi-radios based protocols usually allow the SUs to listening to the control packets exchanges all the time, thus reducing the packet collisions. Moreover, multiple channels can be used simultaneously if multiple data radios are equipped. However, generally speaking, the multi-radios based protocols have higher cost than the single radio based protocols. Although the single radio based protocols have lower cost and power consumption, how to schedule the radio to listen to control packets exchanges and to transmit data packets is a challenging task. In addition, the single radio based protocols are often suffered from the multi-channel hidden terminal problem.

\subsection{Spectrum sensing technique based classification}

Based on the used spectrum sensing techniques, the distributed DSA protocols can be divided into two categories, which are local spectrum sensing based and cooperative spectrum sensing based protocols, respectively. In the local spectrum sensing based protocols, only local sensing results are used to exploit available spectrum opportunities $[20,24,26,27,35,40,50,53,55,56]$. In the latter type of protocols, multiple SUs need to share their local sensing results. The cooperative spectrum sensing can be used either to improve the accuracy of the local sensing results or to collect the whole spectrum information [21-23,25,54].

The local spectrum sensing based protocols design simpler than the cooperative spectrum sensing based protocols. However, some drawbacks are existed. First, local spectrum sensing results may be incorrect due to the impacts of channel fading, shadowing, path loss, etc. Second, it will consume large amount of time to collect the whole spectrum information only based on the local spectrum sensing. Although cooperative spectrum sensing based protocols can efficiently solve above two problems, many challenges need to be solved, e.g., how different SUs exchange their spectrum sensing results, how many SUs should be involved for cooperative sensing, etc. Therefore, the cooperative spectrum sensing based protocol is much more complex than the local spectrum sensing based protocol.

\section{Distributed dynamic spectrum access protocols}

In Section 3, we have classified the existing distributed DSA protocols based on different criteria, such as spectrum sharing modes, spectrum allocation behaviors, spectrum access modes, the usage of common control channel, spectrum usage strategies, the number of radios, and spectrum sensing techniques. In this section, we first survey a large number of state-of-art distributed DSA protocols using different spectrum access modes, which can be categorized into contention-based, slotted, and hybrid protocols. Specifically, the contention-based and slotted distributed DSA protocols will be surveyed in Sections 4.1 and 4.2, respectively, and the hybrid distributed DSA protocols will be reviewed in Section 4.3. Then, we will further discuss our surveyed protocols according to different criteria and properties, such as spectrum sharing modes, sensing techniques, number of radios, cooperation, the usage of common control channel, imperfect sensing, multi-channel hidden terminal problem, channel aggregation, and energy efficiency. Our reviewed distributed DSA protocols contain hardware-constrained cognitive MAC (HC-MAC) [24], stochastic medium access (SMA) Scheme [46], Cognitive Radio-EnAbled Multi-Channel MAC (CREAM-MAC) [50], cognitive MAC (C-MAC) [25], throughput-aimed MAC (T-MAC) [40], opportunistic MAC (O-MAC) [22], polarization-based long-range communication directional MAC (PLRC-MAC) [42], decentralized cognitive MAC (DC-MAC) [35], distributed adaptive opportunistic spectrum access strategy (DA-OSA) [39], dynamic spectrum access MAC (DSA-MAC) [20], dynamic open spectrum sharing MAC (DOSS-MAC) [53], signal-radio adaptive channel MAC (SRAC-MAC) [56], distributed cognitive radio MAC (DCR-MAC) [21], prediction-based dynamic spectrum access (P-DSA) [55], collaborative spectrum sensing based random access protocol (CSS-RA) [54], distributed multi-channel MAC protocol (MMAC-CR) [23], distributed cognitive MAC (COMAC) [43], synchronized MAC (SYN-MAC) [26], dynamic hopping MAC (DH-MAC) [27], pricebased MAC protocol (P-MAC) [41], multi-channel MAC (MC-MAC) [52], and distance-dependent MAC (DDMAC) [44].

\subsection{Contention-based distributed dynamic spectrum access protocols}

The contention-based distributed DSA protocol is one of the most important research area for the distributed DSA protocol design. In [20], Wu et al. proposed a Dynamic Spectrum Access based MAC protocol (DSAMAC) for the distributed CWNs. The DSA-MAC is directly derived from the IEEE 802.11 DCF standard and the common available licensed channels between the source and destination nodes can be determined and reserved through exchanging three control packets on the dedicate CCC. In [53], Ma et al. proposed a dynamic open spectrum sharing MAC (DOSS-MAC) protocol. In the proposed protocol, the spectrum bands are divided into three sub-bands, which are control, data, and busytone bands, respectively. This protocol also tried to 
solve the CCC saturation problem by adopting several measures, such as limiting the traffic on the CCC, adjusting the bandwidth ratio between the $\mathrm{CCC}$ and data band, and allowing the CCC to be migrated to other better bands. In above two protocols, as multiple radios are used, the multi-channel hidden terminal problem can be solved. However, equipping multiple radios for each SU will increase the implementation cost. In order to reduce the implementation cost, a MAC protocol, named signal-radio adaptive channel MAC (SRAC$\mathrm{MAC}$ ), is proposed in [56]. The proposed protocol has three main features: (1) one radio per SU, (2) adaptive channelization which means that multiple fixed channels can be combined to form a new channel, and (3) crosschannel communication which can avoid the interferers and PU activities. However, the SRAC-MAC cannot handle the multi-channel hidden terminal problem. In the distributed CWNs, the hidden PU problem, which is another critically important challenge, is not considered by most existing researches. In order to solve this problem, Yoo et al. proposed a Distributed Cognitive Radio MAC protocol (DCR-MAC) [21]. In the DCR-MAC, while receiving the RTS and the CTS packets, the neighbor nodes of the source and destination nodes will notify the source and destination nodes whether the channels selected by them is occupied by PUs. Through this mechanism, the hidden PU problem can be well solved. However, the throughput and delay of the DCRMAC will be degraded due to the additional control information exchanges.
In [24], a hardware-constrained cognitive MAC (HC$\mathrm{MAC}$ ) protocol is proposed. In this protocol, the maximum number of spectrums that can be used by one SU simultaneously is upper-bounded. The HC-MAC used the theories of sequence decision and optimal stopping to determine the number of sensing channels and maximize the expected throughput. Figure 2 illustrates the intelligent sensing process. Suppose the durations for sensing each channel and transmission are $t$ and $T$, respectively, and all channels have the same bandwidth $B$. As shown in Figure 2a, the immediate reward is $B T$ / $(T+2 t)$ if the node pair transmit immediately after sensing two channels. Then, the immediate reward will be compared with the expected reward for sensing the next channel, denoted by $\mathrm{Ch} 3$. If $\mathrm{Ch} 3$ is not occupied by PUs, the reward will become to $2 B T /(T+3 t)$, as shown in Figure $2 \mathrm{~b}$. Otherwise, only $B T /(T+3 t)$ can be obtained, as shown in Figure 2c. The node pair should determine whether to transmit immediately or sense Ch3 based on the above three possible rewards. When the PU usage statues of different licensed channels are uncorrelated, the $\mathrm{HC}-\mathrm{MAC}$ can realize the optimal throughput. However, if the PU usage statues of different licensed channels are correlated, the HC-MAC can be modified to further improve the network performance. Based on such reason, a novel P-DSA protocol is proposed by Yin et al. [55]. In the P-DSA, the channel correlations are considered, therefore, the number of sensing channels can be reduced through the channel prediction, which can reduce the sensing overhead and

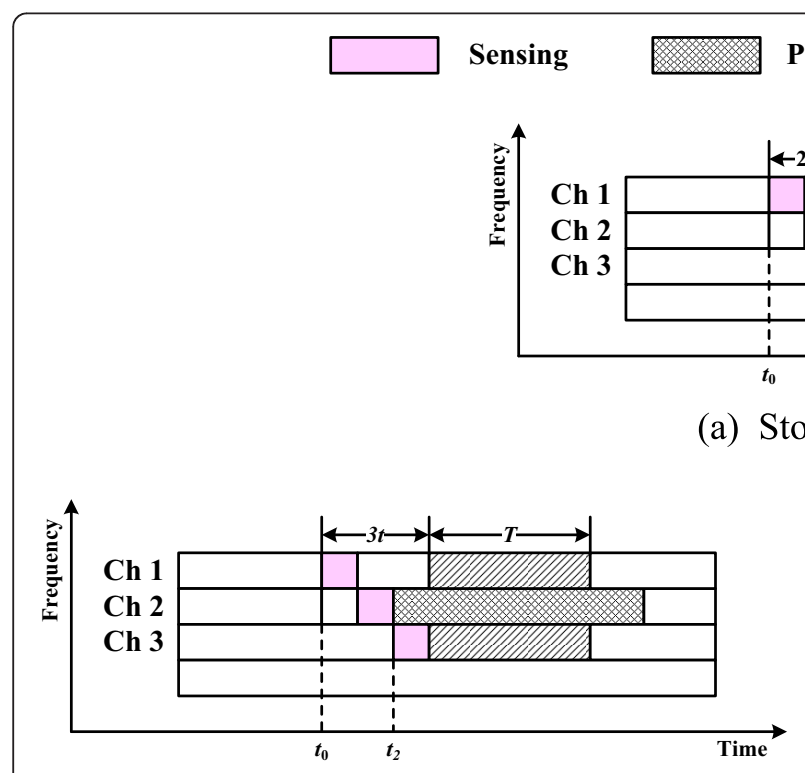

(b) Continue sensing at $t_{2}$ with $\mathrm{Ch} 3$ available

\section{PU occupied TIIIIIS SU transmit}

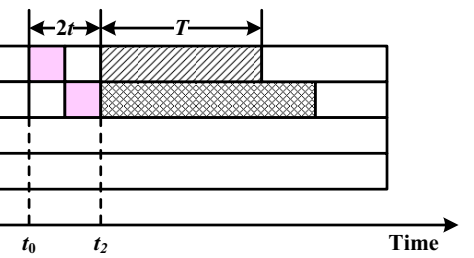

(a) Stop sensing at $t_{2}$

Figure 2 Sketch map for the intelligent sensing process.

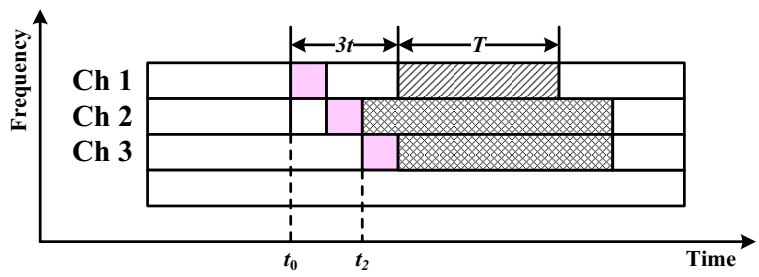

(c) Continue sensing at $t_{2}$ with $\mathrm{Ch} 3$ unavailable 
increase the throughput. However, we must notice that, due to the perfect spectrum sensing, the HC-MAC will not cause the collisions between the SUs and PUs, but such collisions are inevitable in P-DSA because the incorrect prediction is unavoidable. Therefore, a collision threshold, denoted by $\bar{P}_{\text {col }}$, is defined in the PDSA. Figure 3 presents the normalized throughput of the HC-MAC and P-DSA under different numbers of channels. The simulation result shows that the performance of the P-DSA outperforms that of HC-MAC when the channel correlations are taken into consideration.

In the distributed DSA protocols discussed above, the existence of $\mathrm{CCC}$ is the fundamental assumption for protocol designs. However, the predefined CCC may not exist in the realistic distributed CWNs. In order to solve this problem, a novel DSA protocol, named SMA scheme, is proposed by Wang et al. [46]. In the SMA scheme, the source node first uses the Markov-Chain Monte-Carlo (MCMC) method to select a set of channels, denoted as $\Theta$, as the candidate channels for negotiation. The channel selection criterion is to maximize the joint probability of successful negotiations. Then, the RTS packet is sent on the channels in $\Theta$ one by one. If the destination successfully receives the RTS packet, the common available channels for both source and destination can be determined. After the RTS-CTS exchange, the data transmission will be performed on all the common available channels simultaneously. In order to protect PUs' communications and guarantee that different SUs can fairly access channels for transmission, the SMA scheme defines a maximum transmission time, denoted by $T_{\max }$. If the expected transmission time for one node pair is longer than $T_{\max }$, their data packet will be segment into shorter several data fragments (DFs). Figure 4 illustrates the data packet transmission of the SMA scheme. If the DF can be transmitted at a higher rate, more DFs are allowed to be sent until the transmission time exceeds $T_{\max }$. Although the SMA scheme can be used when the CCC is absence, the presence of CCC is still a widely accepted assumption in existing researches. Based on this context, Pawelcazk et al. [19] investigated the performance of the distributed DSA protocols under different kinds of CCC, such as dedicated control channel, hopping control channel, split phase control channel, etc. The authors find that the

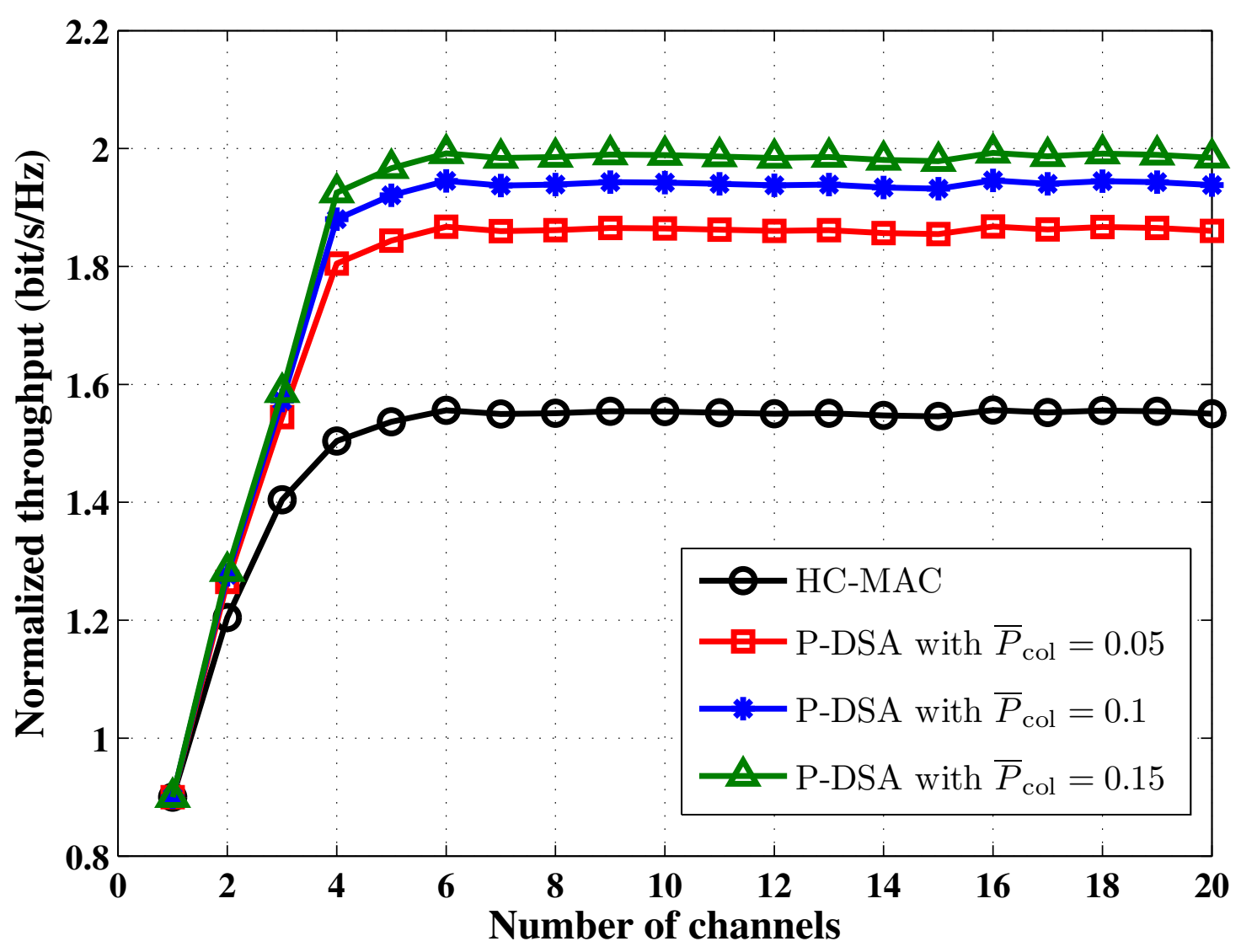

Figure 3 Normalized throughput of the HC-MAC and P-DSA with different numbers of channels and collision thresholds. 


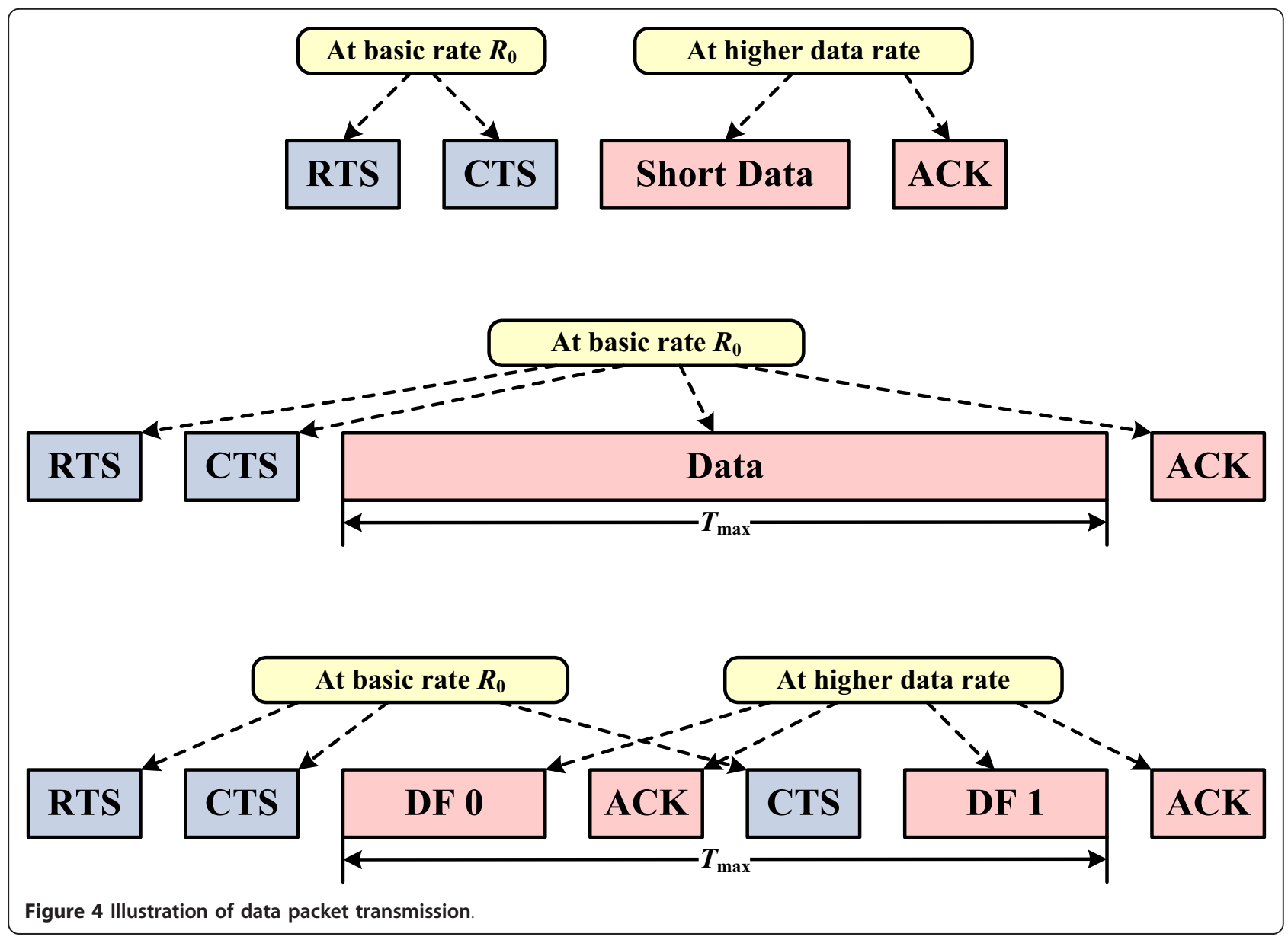

protocol with a dedicated control channel can obtain better performance as the data packet length is increased. Moreover, the protocol with a dedicated control channel, no matter the control channel is licensed to CWNs or not, always outperforms that with hopping control channels.

In cognitive wireless networks, the spectrum sensing outcomes are often unreliable due to the path loss, channel fading, noise uncertainty, shadowing, etc. Therefore, in order to protect PUs, improving the accuracy of sensing results should be combined into the DSA protocol designs. Utilizing spatial and time diversities are two efficient methods to reach the above target. In [54], Chen et al. proposed a CSS-RA, which utilizes the spatial diversity to improve the accuracy of sensing outcomes. In the CSS-RA, the SUs should first sense all channels in the sensing phase. Then, the sensing results of different SUs will be exchanged in the signaling phase. Finally, data transmissions can be performed in the data transmission phase. Through adding the signaling phase between the sensing phase and data transmission phase, the spectrum sensing outcomes will be more accurate. A similar method is also used in the MMAC-
CR [23]. The MMAC-CR is based on the power-saving mode (PSM) of IEEE 802.11 DCF standard. Specifically, this protocol divides each transmission process into two windows, which are ad hoc traffic indication message (ATIM) window and data window, respectively. In the ATIM window, all SUs will first fast scan all channels and obtain the corresponding spectrum statues. Then, SUs will share their spectrum sensing results by sending busy signals during the mini-frame contained in each ATIM window. The fusion rule used by MMAC-CR is the OR rule. In [50], a CREAM-MAC protocol is proposed by Zhang et al. Different from the CSS-RA and MMAC-CR, the CREAM-MAC utilizes the time diversity to improve the accuracy of sensing results. Specifically, if the current sensing result satisfies the predefined accuracy, the SUs will stop spectrum sensing and perform data transmissions. Otherwise, one more sensing process will be implemented to improve the accuracy of sensing result.

In most of existing studies, spectrum sensing plays an critically important role in the protocol design. However, some researchers try to realize dynamic spectrum sharing without using spectrum sensing. In [43], 
Salameh et al. proposed a novel protocol, named COMAC. The COMAC can provide soft guarantees for PUs without using spectrum sensing. Specifically, SUs first calculate two kinds of interferences, which are PRto-CR interference and PR-to-PR interference, respectively. Then, the transmit power can be dynamically adjusted to guarantee that the outage probability of PUs satisfies the pre-defined threshold. Another method to protect PUs without spectrum sensing is to utilize the power mask, which defines the maximal transmit power of SUs. such as the DDMAC [44] and P-MAC [41]. In the DDMAC, in order to more efficiently utilize spectrum resources, a distance-dependent channel assignment algorithm is proposed. The basic idea of this algorithm is to assign the channel with lower SINR to the source-destination pair with shorter distance. Through such cooperative channel assignment, the number of simultaneous transmissions can be significantly increased. Game theory is another method to perform distributed resource allocation and increase the number of simultaneous transmissions. In [57], Wang et al. proposed the P-MAC. In this protocol, a price-based iterative water-filling (PIWF) algorithm is proposed and the authors proved that the proposed PIWF algorithm can reach a good Nash equilibrium. In order to implement above PIWF algorithm, the P-DSA divides each slot into two windows, which are contention window (CW) and data window (DW), respectively, and each $\mathrm{CW}$ is further divided into two sub-windows, named access window (AW) and training window (TW), respectively. In the AW, multiple source-destination pairs compete for accessing the $\mathrm{CCC}$ by exchanging RTS, CTS, and DTS packets. In the TW, those pairs that successfully exchanged control packets can iteratively negotiate their transmission parameters in a TDMA manner. Finally, multiple source-destination pairs will finish their data transmissions simultaneously in the DW.

\subsection{Slotted distributed dynamic spectrum access protocols}

As described in Section 3.3, compared with contentionbased protocols, slotted DSA protocols can obtain better network performances, such as higher network throughput and lower access delay, due to the stringent time management. One of the most famous slotted distributed DSA protocol is the cognitive MAC (C-MAC) protocol [25], which is based on the well-known IEEE 802.22 standard. In the C-MAC, a rendezvous channel, which can be viewed as the best channel under current network status, is dynamically selected. The rendezvous channel acts as the CCC and can be moved to other channels when the network status changes. For each channel, the identical super frame is defined. Each super frame contains one beacon period, quiet period, and data transfer period, respectively. In the quiet period, all SUs on the same channel must stop transmitting and perform spectrum sensing. In the beacon period, each SU will be allocated a unique beacon slot and the information of each SU will be broadcast in the corresponding beacon slot. Then, the data transmissions can be performed in the data transfer period according to the schedules in the previous beacon period. Moreover, the beacon and quiet periods of different channels are nonoverlapping based on the inter-channel coordination mechanism, such that the SUs staying on one certain channel can perform out-of-band measurements and obtain the traffic load information of other channels. Consequently, each SU can choose to quit current channel and join another channel with lower traffic loads and thus the total traffic loads of the entire network can be evenly distributed on all available channels.

In [40], a novel slotted DSA protocol named T-MAC protocol is proposed. The super frame structure of $\mathrm{T}$ MAC is shown in Figure 5. Each super frame consists of one reservation frame, $K$ information frames and one acknowledgment frame. Each reservation frame contains one sensing slot and $N$ reservation slots. Correspondingly, each information frame and acknowledgment frame consists of $N$ information slots and acknowledgment slots. In order to decide which channel is available in current slot, all nodes must sense data channels in sensing slots. Each reservation slot is dedicated to the reservation of the corresponding information slot and acknowledgment slot. Each reservation period is a reservation process implemented through three-phase dialogues, named reservation request (RR), reservation confirmation (RC) and deciding to send (DTS). Setting several reservation periods in one reservation slot is to enhance the reservation success rate, which can facilitate concurrent transmissions. In the T-MAC, data packets and ACK packets can be protected simultaneously through the special acknowledgment frames. An efficient power control mechanism is also provided, which can significantly increase the space reuse efficiency. Moreover, the negative impact caused by the multichannel hidden terminal problem can be efficiently reduced. In addition, the multi-channel exposed terminal problem is also defined in this investigation and the authors explained that this problem can also be solved. Figures 6 and 7 present the network throughput and access delay of the T-MAC and DSA-MAC [20], respectively. We can observe from the simulation results that the T-MAC obviously outperforms the DSA-MAC.

4.3 Hybrid distributed dynamic spectrum access protocols The hybrid protocols aim to obtain a tradeoff between the contention-based protocols and slotted protocols. 


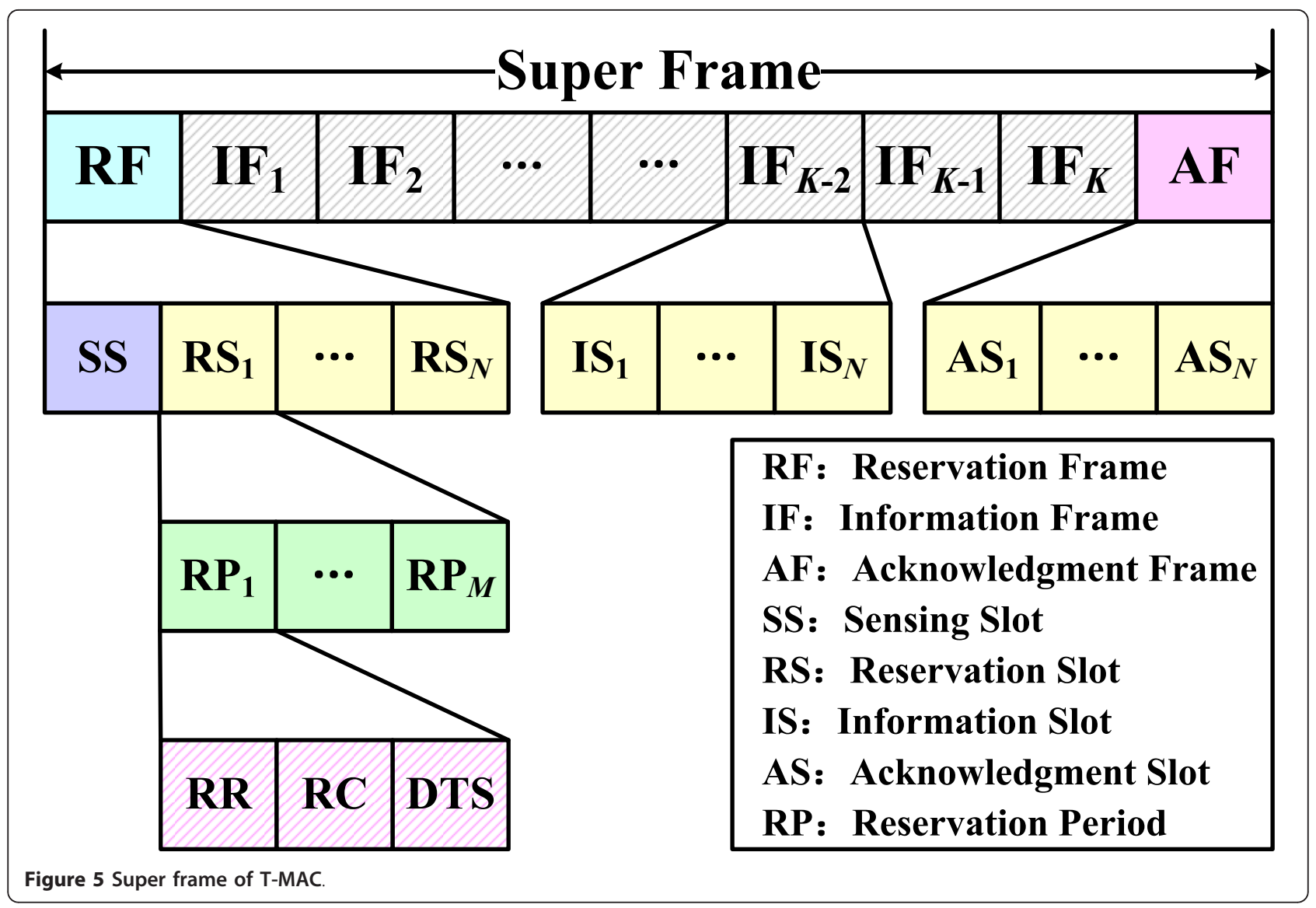

Usually, the hybrid DSA protocol divides time axes into synchronized slots. In each slot, SUs compete for transmission through contention-based methods. The performance of the hybrid protocols are usually better than that of contention-based protocols and the complexity is lower than that of slotted protocols.

$\mathrm{Su}$ and Zhang proposed an opportunistic MAC protocol (O-MAC) for distributed CWNs [22]. The protocol integrates the spectrum sensing, which is performed in the physical layer, and the packet scheduling implemented at MAC layer. In the O-MAC, each slot includes two phases, namely, the reporting phase and negotiating phase, and the former phase is further divided into a series of mini-slots. In the reporting phase, spectrum sensing information are broadcasted by all SUs based on their own local observations. In the negotiating phase, the node pair, which successfully exchanged control packets, can use all un-occupied channels for data transmission. In order to improve the spectrum sensing efficiency, two sensing policies, which are random sensing policy and negotiation-based sensing policy, respectively, are proposed. In order to further increase the network throughput, Iyer et al. proposed a novel multi-channel MAC (MC-MAC) protocol, which integrates spectrum sensing, packet scheduling, and channel allocation [52]. In the MC-MAC, the channel allocation problem is solved based on the Weighted Bipartite Graph Matching technique and three channel allocation schemes, which are Random node matching, Maximum utility matching, and Max-Min reward, respectively, are investigated. Figure 8 presents the network throughput of the O-MAC and MC-MAC. Although the MC-MAC can achieve higher network throughput than the O-MAC, the channel allocation in the MC-MAC is performed by a spectrum broker, which may not exist in realistic distributed CWNs.

In [26], Kondareddy and Agrawal proposed a SYNMAC protocol, in which the dedicated CCC is not needed. In the SYN-MAC, all SUs share the same frequency hopping sequence and two radios, which are listening radio and data radio, respectively, are equipped for each SU. Each SU will switch its listening radio according to the pre-defined frequency hopping sequence to exchange and overhear control packets. Once one channel is successfully reserved, the source and destination nodes will finish their data transmission on the reserved channel using their data radios. Moreover, the multi-channel hidden terminal problem can be 


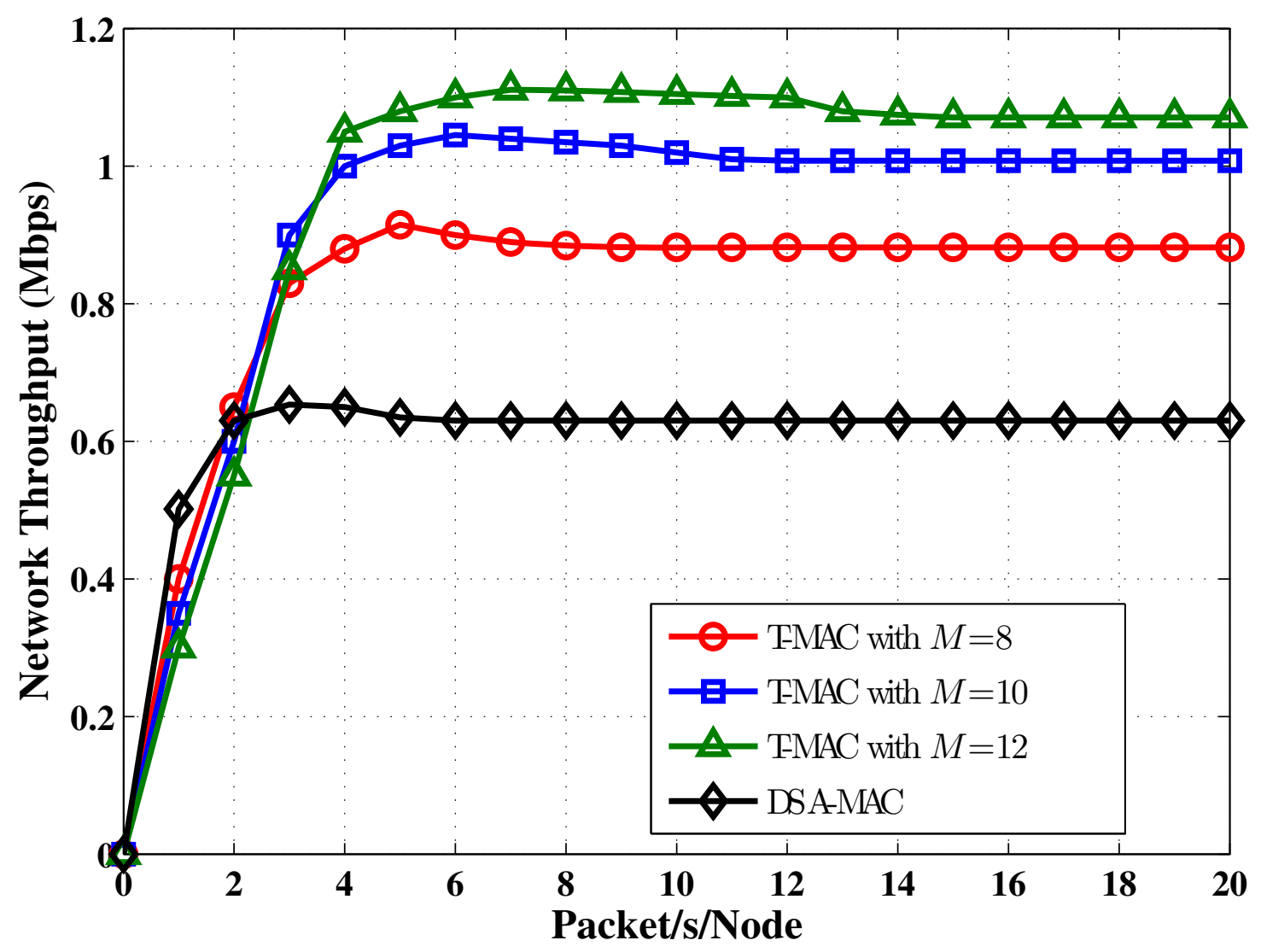

Figure 6 Network throughput of T-MAC and DSA-MAC as the function of traffic load.

solved because all SUs are synchronized and the listening radios of different SUs are always on the same channel due to the same hopping sequence. Although no dedicate CCC is needed in the SYN-MAC, CCC saturation problem is still existed because all control packets are exchanged on the same channel. In order to solve this problem, a novel MAC protocol, named dynamic channel hopping MAC (DH-MAC), is proposed in [27]. Instead of use the same frequency hopping sequence, each SU will generate its unique frequency hopping sequence and frequency hopping sequences of neighbor nodes can be obtained by the broadcast mechanism. In this way, different node pairs can exchange their control packets on different channels, thus efficiently alleviating the CCC saturation problem. Moreover, only one radio is needed for each SU. However, we need notice that, although the multi-channel hidden terminal problem cannot be fully settled, the probability that the multichannel hidden terminal problem happens is small. Figure 9 shows the network throughput of SYN-MAC and DH-MAC as the function of the number of SUs. Because the CCC saturation problem still exists in the SYN-MAC but is well solved in DH-MAC, the perfor- mance of DH-MAC is significantly improved especially when the number of SUs is large.

In most of existing DSA protocols, only omnidirectional antenna is assumed. Although using antenna technologies (such as directional antenna and polarized antenna, etc.) is an efficient way to improve the network performance and large number of studies have been researched for traditional wireless networks, rare investigations have been done for CWNs. In [42], Wang et al. proposed a polarization-based long-range communication directional MAC protocol (PLRC-DMAC) for distributed cognitive wireless networks, in which the directional antenna and antenna polarization characteristic are considered simultaneously. The PLRC-DMAC used ON/OFF model to characterize the channel usage and polarization states of PUs. Through utilizing polarized antenna, available resources for SUs are increased. Figure 10 illustrates the ON/OFF of this protocol. If antenna polarization is not considered, once some particular channels are occupied by PUs, these channels cannot be used by SUs for the corresponding slots, such as channel 1 are not available for SUs during slot 1 and $i$ and channel 2 cannot used by SUs during slot 2 and $i$. 


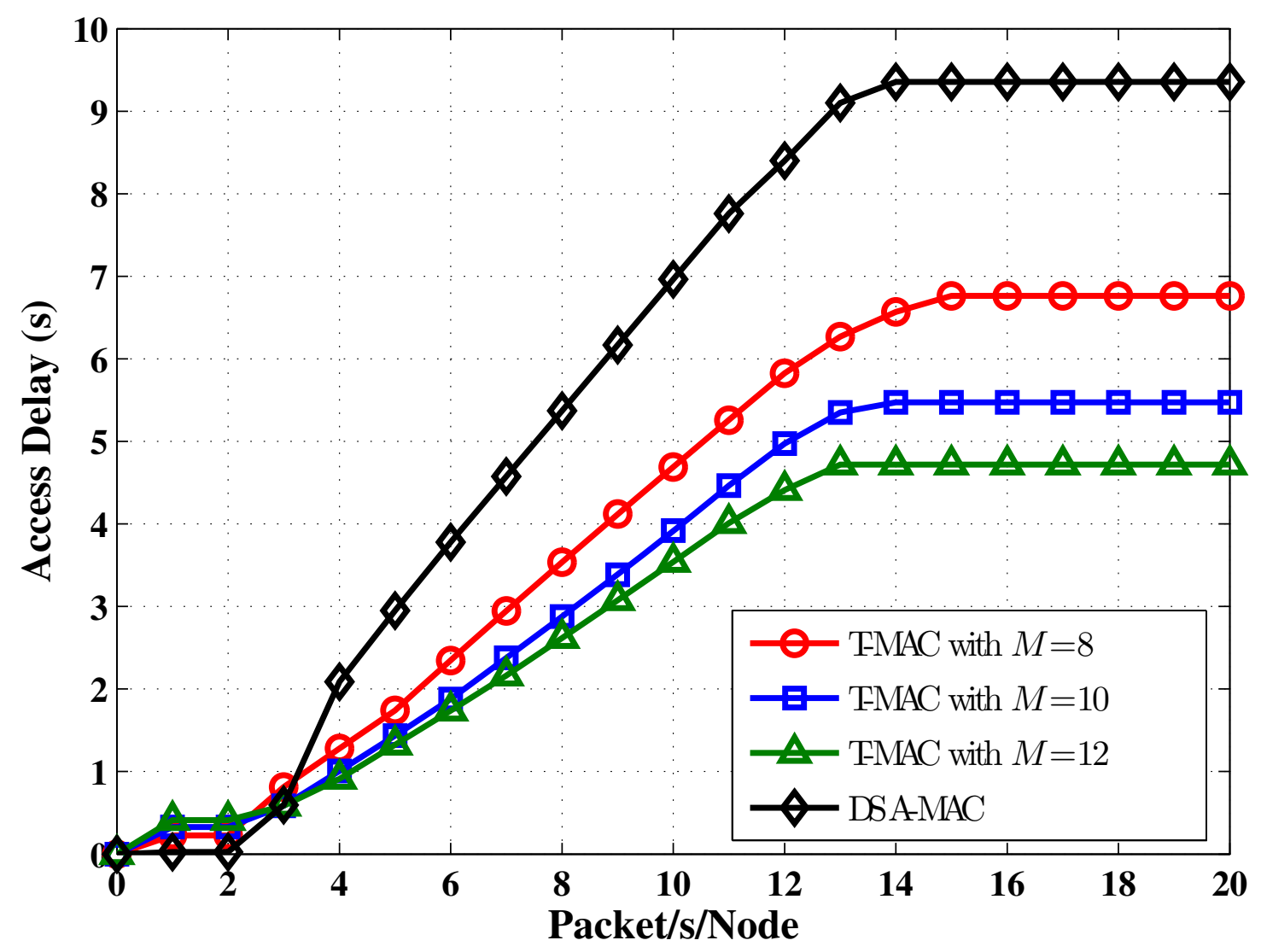

Figure 7 Access delay of T-MAC and DSA-MAC as the function of traffic load.

However, when we utilizes polarized antenna, these channels can also be used by SUs, such as SUs can transmit on channel 1 with vertical polarization during slot 1 . In addition, directional antenna is used to establish long-range communication links, through which two one-hop transmissions can be replaced by a onehop long-range transmission, and the authors proved that such long-range communication links can efficiently increase the spatial reuse efficiency of spectrum resources.

Intelligence and learning ability are two important features of CWNs and full-spectrum sensing usually cannot be implemented in reality. Based on this context, a novel DSA protocol, named DC-MAC, is proposed [35]. The DC-MAC is based on the partial observable Markov decision process (POMDP) framework and each $\mathrm{SU}$ is allowed to sense only one channel in each slot. In order to maximize the total throughput over finite slots, at the beginning of each slot, the SU needs to choose which channel will be sensed based on current belief vector, which is a sufficient statistic to describe the current states of all channels. Based on the pioneering study, several investigations have been conducted. In $[36,58]$, the myopic policy, in which the SU only aims to maximize the throughput in current slot, is established and the optimality of myopic policy is proved. In [34], the spectrum sensor, sensing strategy, and access strategy are jointly designed and the objective is to maximize the throughput of the SU while guaranteeing that the collision probability cannot exceed the predefined threshold. Moreover, a separation principle is proposed, which can efficiently reduce the complexity. In $[37,38]$, the DSA strategies with energy constraint are investigated. In these studies, the threshold structures of optimal strategies are also established. In the above researches, only one channel or fixed number of multiple channels are assumed to be sensed during each slot, which cannot fully reflect the adaptive feature of CWNs and causes that SUs are easily affected by PUs. Based on this context, a distributed adaptive opportunistic spectrum access strategy (DA-OSA) is proposed [39]. In the DA-OSA, multiple channels are allowed to be sensed in each slot and the number of sensing channels in each slot is dynamically determined. Specifically, at the beginning of each slot, the SU first chooses a channel to sense based on the prior information for the spectrum access of PUs. Then, based on the sensing result, the immediate reward will be compared with the maximal 


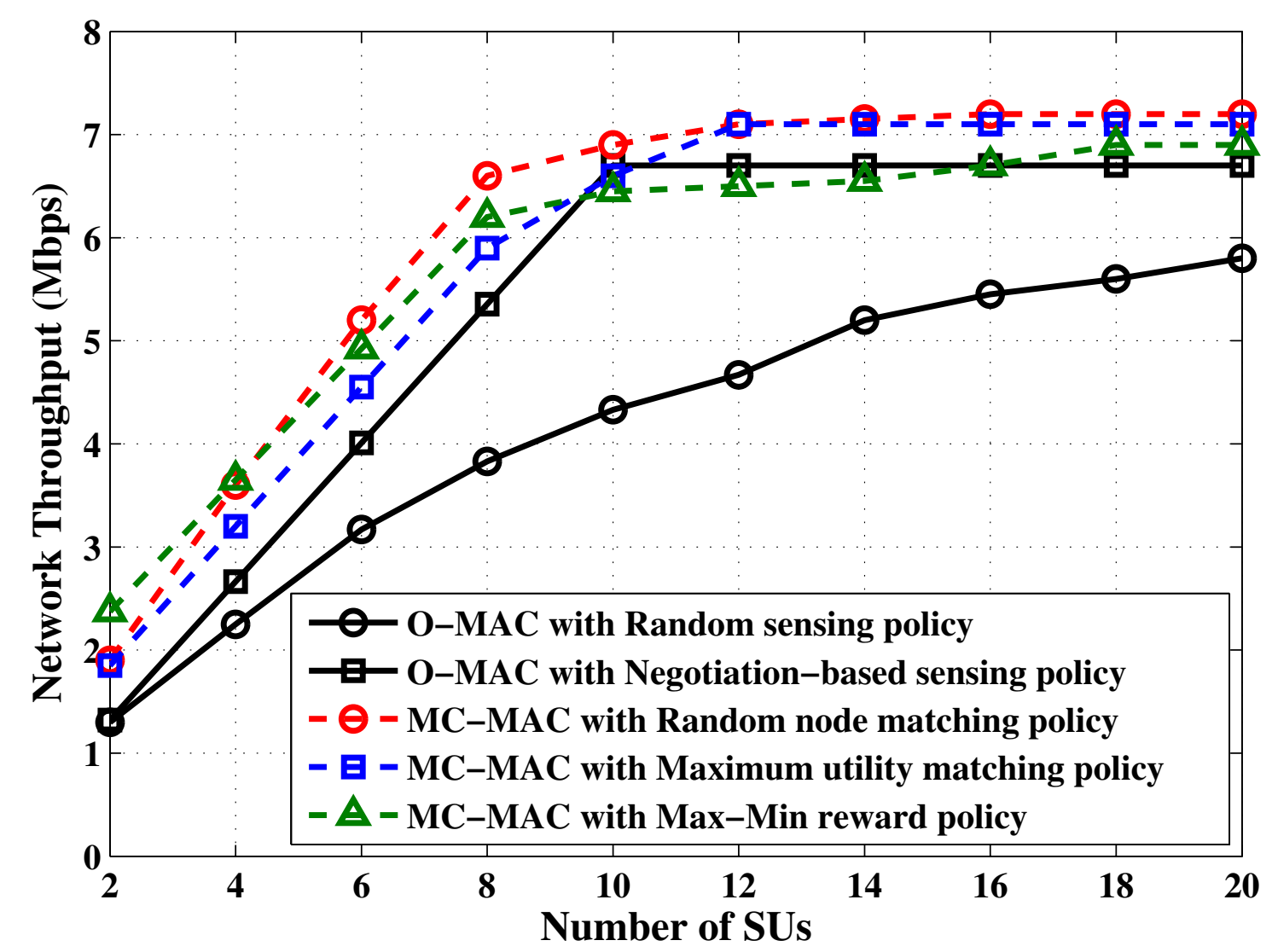

Figure 8 Network throughput of O-MAC and MC-MAC as the function of the number of SUs.

expected reward. If the former reward is lager than the latter one, the SU will stop sensing and transmit data packets immediately. Otherwise, the channel, which can maximize the expected reward, is selected based on the current belief vector and the sensing results of the sensed channels for further sensing.

\subsection{Further discussions}

In Section 4.1, 4.2, and 4.3, we have investigated a large number of state-of-art distributed DSA protocols based on different spectrum access modes. All discussed protocols and their characteristics are listed in Table 1. Now we will further discuss these protocols according to different metrics.

Based on Table 1, we can observe that most of existing distributed DSA protocols belong to the contentionbased protocols and hybrid protocols, only T-MAC [40] and C-MAC [25] are slotted protocols. The reason for such phenomenon is that the design of slotted protocols is more complex than the design of contention-based and slotted protocols. However, generally speaking, the slotted protocols can realize better performance than the other kinds of protocols. As shown in Figures 6 and 7 , compared with the DSA-MAC [20], which belongs to the contention-based protocol, the throughput and delay of T-MAC are significantly improved.

Most of our investigated protocols reserve channels for data transmissions similar with the well-known IEEE 802.11 DCF standard, such as DSA-MAC [20], DCRMAC [21], HC-MAC [24], CREAM-MAC [50], O-MAC [22], SYN-MAC [26], COMAC [43], DDMAC [44], etc. In these protocols, the RTS and CTS packets are used for channel reservation. Although some additional control packets are used for different purposes, such as avoiding the collisions between the SUs and PUs [50] and collecting spectrum sensing information from neighbor SUs [21], the channel reservation is still performed as the handshake mechanism defined in the IEEE 802.11 DCF standard.

As discussed in Section 3.2, the existing distributed DSA protocols can be divided into cooperative and noncooperative protocols according to different spectrum allocation behaviors. As shown in Table 1, most protocols perform spectrum allocation without cooperation, such that each source-destination pair only aims to maximize their own performance. This kind of protocols usually has simple architectures. Compared with noncooperative protocols, cooperative protocols often try to 

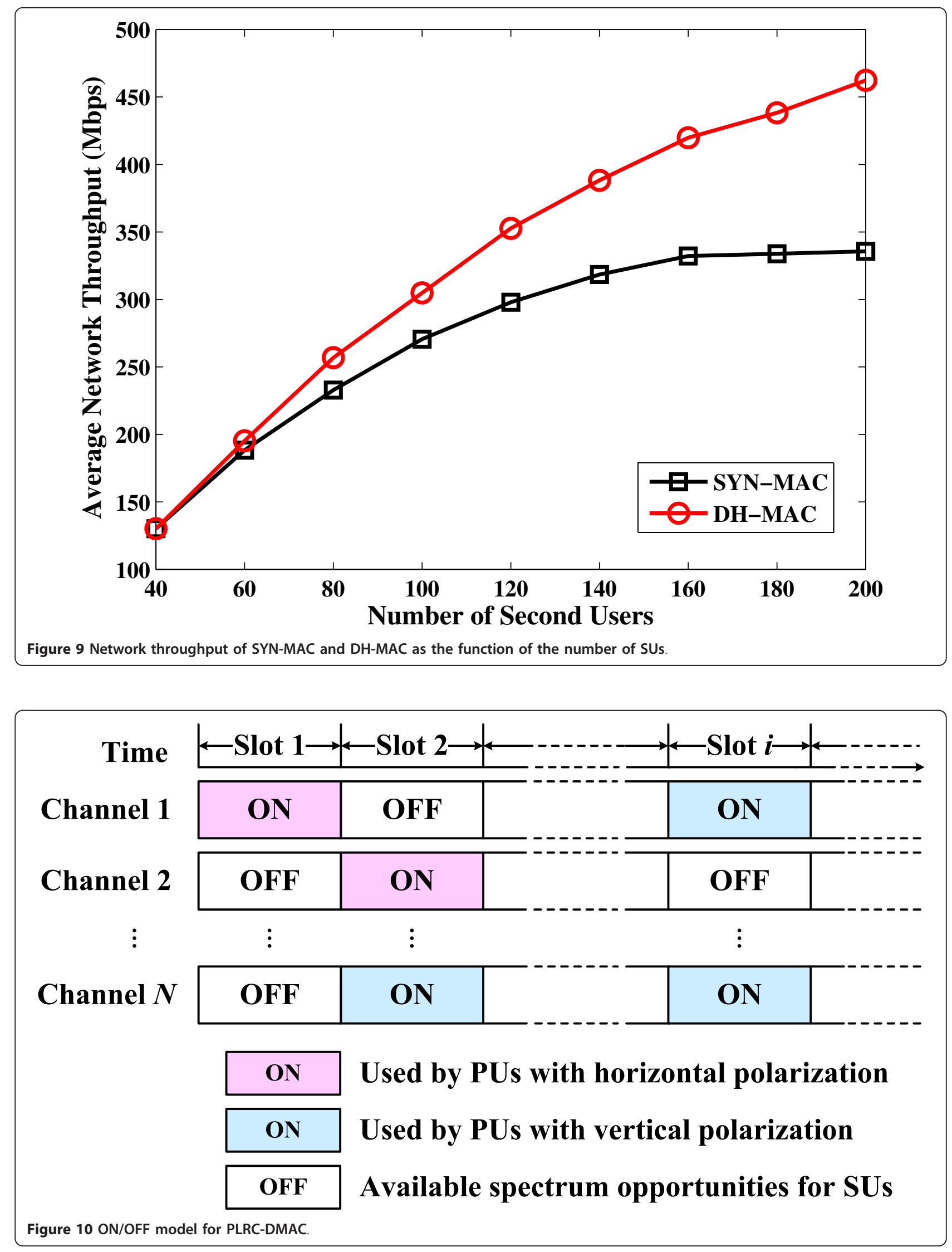
Table 1 Characteristics of discussed distributed DSA protocols

\begin{tabular}{|c|c|c|c|c|c|c|c|c|c|c|}
\hline Protocol & $\begin{array}{l}\text { Spectrum } \\
\text { access } \\
\text { mode }\end{array}$ & Cooperation & $\begin{array}{l}\text { Spectrum } \\
\text { sharing } \\
\text { mode }\end{array}$ & $\begin{array}{l}\text { Dedicated } \\
\text { CCC }\end{array}$ & $\begin{array}{l}\text { Sensing } \\
\text { technique }\end{array}$ & $\begin{array}{l}\text { Imperfect } \\
\text { sensing }\end{array}$ & $\begin{array}{l}\text { Number } \\
\text { of } \\
\text { radios }\end{array}$ & $\begin{array}{l}\text { Multi-channel } \\
\text { hidden terminal } \\
\text { problem }\end{array}$ & $\begin{array}{l}\text { Channel } \\
\text { aggregation }\end{array}$ & $\begin{array}{l}\text { Energy } \\
\text { efficiency }\end{array}$ \\
\hline $\begin{array}{l}\text { DSA- } \\
\text { MAC }\end{array}$ & Contention & No & Overlay & $\begin{array}{l}\text { Yes, } \\
\text { Licensed }\end{array}$ & Local & No & 2 & No & No & No \\
\hline $\begin{array}{l}\text { DOSS- } \\
\text { MAC }\end{array}$ & Contention & No & Overlay & $\begin{array}{l}\text { Yes, } \\
\text { Licensed }\end{array}$ & Local & No & 3 & No & No & No \\
\hline $\begin{array}{l}\text { SRAC- } \\
\text { MAC }\end{array}$ & Contention & No & Overlay & No & Local & No & 1 & Yes & Yes & No \\
\hline $\begin{array}{l}\text { DCR- } \\
\text { MAC }\end{array}$ & Contention & No & Overlay & $\begin{array}{l}\text { Yes, } \\
\text { Licensed }\end{array}$ & Cooperative & No & 2 & No & No & No \\
\hline HC-MAC & Contention & No & Overlay & $\begin{array}{l}\text { Yes, } \\
\text { Licensed }\end{array}$ & Local & No & 1 & No & Yes & No \\
\hline P-DSA & Contention & No & Overlay & $\begin{array}{l}\text { Yes, } \\
\text { Licensed }\end{array}$ & Local & No & 1 & No & Yes & No \\
\hline SMA & Contention & No & Overlay & No & Local & Yes & 1 & Yes & Yes & No \\
\hline CSS-RA & Contention & No & Overlay & $\begin{array}{l}\text { Yes, } \\
\text { Licensed }\end{array}$ & Cooperative & No & 1 & Yes & No & No \\
\hline $\begin{array}{l}\text { CREAM- } \\
\text { MAC }\end{array}$ & Contention & No & Overlay & $\begin{array}{l}\text { Yes, } \\
\text { Licensed }\end{array}$ & Local & Yes & 1 & No & Yes & No \\
\hline C-MAC & Slotted & No & Overlay & $\begin{array}{l}\text { Yes, } \\
\text { Unlicensed }\end{array}$ & Cooperative & No & 1 & No & No & No \\
\hline T-MAC & Slotted & No & Overlay & $\begin{array}{l}\text { Yes, } \\
\text { Licensed }\end{array}$ & Local & No & 1 & No & No & Yes \\
\hline O-MAC & Hybrid & No & Overlay & $\begin{array}{l}\text { Yes, } \\
\text { Licensed }\end{array}$ & Cooperative & No & 2 & No & Yes & No \\
\hline MC-MAC & Hybrid & Yes & Overlay & $\begin{array}{l}\text { Yes, } \\
\text { Licensed }\end{array}$ & Local & No & 2 & No & Yes & No \\
\hline $\begin{array}{l}\text { SYN- } \\
\text { MAC }\end{array}$ & Hybrid & No & Overlay & No & Local & No & 2 & No & No & No \\
\hline DH-MAC & Hybrid & No & Overlay & No & Local & No & 1 & Yes & No & No \\
\hline $\begin{array}{l}\text { PLRC- } \\
\text { DMAC }\end{array}$ & Hybrid & No & Overlay & $\begin{array}{l}\text { Yes, } \\
\text { Licensed }\end{array}$ & Local & No & 2 & No & No & Yes \\
\hline DC-MAC & Hybrid & No & Overlay & No & Local & Yes & 1 & No & No & No \\
\hline DA-OSA & Hybrid & No & Overlay & No & Local & No & 1 & No & Yes & No \\
\hline P-MAC & Contention & Yes & Underlay & $\begin{array}{l}\text { Yes, } \\
\text { Licensed }\end{array}$ & No & No & 2 & No & Yes & Yes \\
\hline $\begin{array}{l}\text { MMAC- } \\
\text { CR }\end{array}$ & Contention & No & Overlay & $\begin{array}{l}\text { Yes, } \\
\text { Licensed }\end{array}$ & Cooperative & No & 2 & No & No & Yes \\
\hline COMAC & Contention & No & Underlay & $\begin{array}{l}\text { Yes, } \\
\text { Licensed }\end{array}$ & No & No & $>2$ & No & No & Yes \\
\hline DDMAC & Contention & Yes & Underlay & $\begin{array}{l}\text { Yes, } \\
\text { Licensed }\end{array}$ & No & No & $>2$ & No & No & Yes \\
\hline
\end{tabular}

optimize the network performance by solving complex optimization algorithms, such as MC-MAC [52], PMAC [41], and DDMAC [44]. Although the architecture of the cooperative protocol is more complex than that of the non-cooperative protocol, the former one generally outperforms the latter one. For example, as shown in Figure 8, the MC-MAC, in which the spectrum allocation is performed with cooperative method, can achieve better network throughput than the O-MAC [22].

Overlay and underlay are two fundamental spectrum sharing modes for the CWNs. From the Table 1, we can observe that most of our discussed protocols belong to the overlay mode, in which the spectrum sensing is one of the most important functions for SUs to exploit the opportunistic spectrum bands that are not occupied by PUs. In our investigated protocols, the P-MAC [41], COMAC [43], and DDMAC [44] belong to the underlay mode. In the P-MAC and DDMAC, the maximum transmit power for SUs named power mask is pre-defined for each channel. In this way, the PUs' transmissions will not be interrupted by SUs. Different from P-MAC and DDMAC, the COMAC does not use the pre-defined power mask to limit the transmit power of SUs. In the COMAC, SUs can estimate the interferences suffered by PUs, thus dynamically adjusting their transmit power. 
Using a dedicated CCC for exchanging control packets is widely accepted by researchers. Most of the existing protocols are also built upon the existence of the dedicated CCC. Based on our survey, we find that most protocols assume the dedicated CCC is licensed for CWNs such that the control packets exchanges of SUs are interference-free from the PUs. Only the C-MAC [25] uses the unlicensed channel as the dedicated CCC. As investigated in [19], the performance with unlicensed CCC is worse than that with licensed CCC because PUs' transmissions may block the unlicensed CCC for long time. In our discussions, the SRC-MAC [56], SMA [46], SYN-MAC [26], and DH-MAC [27], etc. do not rely on the dedicated CCC.

As discussed before, spectrum sensing is one of the most important functions for the CWNs to obtain the available opportunistic spectrum bands. From the Table 1 , we can observe that most of the existing protocols only allow each SU to use its local spectrum sensing to derive spectrum opportunities. Although only utilizing local sensing can simplify the protocol architecture, it can also lead to some problems, such as sensing errors and hidden PU problem. Cooperative sensing is an efficient method to solve these problems. In DCR-MAC [21], the cooperative sensing is used for solving the hidden PU problem. In CSS-RA [54], sensing errors are reduced by cooperative sensing. In O-MAC [22] and MMAC-CR [23], the cooperative sensing is used to help each SU obtain the whole spectrum opportunity map.

In the existing researches, most protocols assume that SUs can derive correct sensing results. However, due to the impacts of fading, noise uncertainty, and shadowing, SUs may often obtain incorrect sensing results. CSS-RA [54] and CREAM-MAC [50] provide two efficient methods to improve spectrum sensing accuracy. Compared with the CSS-RA, in which the cooperative spectrum sensing is used, the CREAM-MAC utilizes time diversity to increase the sensing accuracy, i.e., each SU can perform spectrum sensing in multiple slots.

Many DSA protocols assume that each SU are equipped with multiple radios. In these protocols, one radio is often dedicated for transmitting, receiving, and overhearing control packets. For example, DSA-MAC [20], DCR-MAC [21], O-MAC [22], etc., all use one radio to work on the dedicated CCC and the SYN-MAC [26] dynamically switches the control radio according to the pre-defined frequency hopping sequence for control packets exchanges. Utilizing multiple radios can also increase the network throughput, such as COMAC [43] and DDMAC [44]. In this protocols, multiple data radios are used, thus each $\mathrm{SU}$ can perform its data transmissions on multiple channels simultaneously.

Multi-channel hidden terminal problem is critically important to CWNs. Therefore, many protocols try to solve this problem. Using one control radio to dedicated overhear control packets is a widely accepted method to overcome this problem, such as DSA-MAC [20], DCRMAC [21], O-MAC [22], SYN-MAC [26], COMAC [43], DDMAC [44], and so on. Other methods can also be used. For example, the CREAM-MAC [50] only allows the $\mathrm{SU}$, which just finished the data transmission, can only reserve the same channels that are used during the last transmission within a pre-defined interval. In this way, although only one radio is equipped for each SU, the multi-channel hidden terminal problem can be solved.

In realistic CWNs, the available spectrum bands that are not occupied by PUs may be discontinuous. In order to use such discontinuous channels simultaneously to increase the network throughput, multiple data radio can be utilized with each one dedicated working on one channel, such as the COMAC [43] and DDMAC [44]. Another method is to use channel aggregating technique, which allows one radio to operate on multiple discontinuous spectrum bands simultaneously (e.g., orthogonal frequency division multiplexing access (OFDMA)). Therefore, many protocols use the channel aggregating technique to implement multi-channel operation, such as the CREAM-MAC [50] and HCMAC [24].

Energy efficiency is a main concern in the CWNs. Power control mechanism is one efficient method to improve the energy efficiency, such as P-MAC [41], PLRC-MAC [42], T-MAC [40], etc. In these protocols, the transmit power of SUs are dynamically adjusted, which can efficiently reduce the mutual interferences among SUs, increase the network throughput, and improve energy efficiency. Another method for improving energy efficiency is used by the MMAC-CR [23]. In this protocol, the SUs which do not successfully reserve channels can go into the sleeping mode, which can efficiently save energy. Moreover, the MMAC-CR can perform two sensing schemes, which are fast sensing and fine sensing, respectively. The SUs can choose fast sensing to improve the energy efficiency.

\section{Cognitive radio standards}

In recent years, lots of efforts are conducted to create international CR standards. These standards aim to utilize the TV White Space (TVWS) by using CR technology. In this section, we will briefly introduce some famous CR standards, such as IEEE 802.22, Ecma 392, IEEE 802.11 AF, IEEE 802.19, and IEEE SCC 41.

IEEE 802.22 standard is the first world-wide CR standard for the wireless regional area network (WRAN) [1,2]. The IEEE 802.22 standard not only specifies the $\mathrm{PHY}$ and MAC protocols, but also includes many cognitive functions, such as spectrum management, sensing 
interface, geo-location and database access, etc. This standard aims to provide broadband wireless access in the TVWS and the data transmission is designed to be $1.5 \mathrm{Mb} / \mathrm{s}$ for the downlink and $384 \mathrm{~kb} / \mathrm{s}$ for the uplink.

Ecma 392 standard is the first CR standard which aims to develop the communication standard for personal/portable devices to exploit the TVWS [3,4]. Ecma 392 specifies the PHY and MAC protocols for personal/ portable devices. Adaption to worldwide regulatory requirements and robust support for real-time traffic are two main characteristics of Ecma 392. Unlike the infrastructured network in IEEE 802.22 standard, two kinds of network architectures are supported by Ecma 392, which are master-slave and peer-to-peer networks, respectively. In the master-to-slave network, the dynamic frequency selection (DFS), transmit power control (TPC) and channel measurement are conducted by the master. However, these functions described above are coordinated by peer devices with a distributed manner in the peer-to-peer network.

Another CR standard that allows personal/portable devices to dynamically access the TVWS is the IEEE 802.11 AF standard [5]. Because the spectrum bands licensed to TVWS have better propagation characteristics than the ISM bands, this standard is expected to provide higher data transmission rate than current IEEE 802.11 standards. In the IEEE 802.11 AF standard, the access point (AP) is embedded with CR function and can dynamically obtain the available spectrum bands in the TVWS according to the specific spectrum trading schemes.

Besides the IEEE 802.22, Ecma 392, and IEEE 802.11 AF standards, some new CR standards are exploiting, such as IEEE 802.19 and IEEE SCC 41 standards [3]. Because developing CWNs may cause the interferences between heterogeneous CR systems, these two standards aim to such problem. Specifically, IEEE 802.19 standard is used to guarantee the coexistence of multiple heterogeneous CWNs that will operate in the TVWS. The main contribution of IEEE SCC 41 standard is that it defines higher layer (higher than PHY and MAC layers) standards for CWNs.

\section{Open research issues}

Prior to this section, a technology survey for DSA protocols of distributed CWNs has been provided. In this section, we explore the opportunities and open research issues for future investigation of distributed DSA protocols.

\subsection{Spectrum handoff based distributed dynamic spectrum access protocols}

In most of existing distributed DSA protocols, ON/OFF model is used. In this model, the statistics of channel usage for PUs remain unchanged during each slot. However, PUs may access the licensed spectrum used by SUs at any time. In this case, in order to prevent the ongoing transmissions of SUs from outage, the spectrum handoff mechanism should be integrated into the DSA protocols. Unlike the centralized CWNs, in which the backup spectrum can be designated by central controllers, the spectrum handoff mechanism for distributed CWNs is more complex and should include several important components, such as distributed backup spectrum selection, spectrum handoff coordination, transmission recovery, etc.

\subsection{Spectrum prediction based distributed dynamic spectrum access protocols}

Intelligence and learning ability are two important features of the CWNs. However, most existing DSA protocols do not take full advantage of these two characteristics. Using the intelligence and learning ability to perform spectrum prediction could efficiently improve the performance of distributed CWNs. First, SUs can obtain which channel is the most likely to be unoccupied through spectrum prediction and sense the corresponding channel, which can reduce the sensing overhead. Second, SUs can choose the backup channel through predicting which channel will be available, thus supporting the spectrum handoff. Third, each SU can predict the channel with lower traffic and access the corresponding channel, which could reduce the packet collisions. Therefore, the spectrum prediction based distributed dynamic spectrum access protocols are worth for further investigation.

\subsection{Adaptation of the spectrum-sharing modes}

Overlay and underlay are two available spectrum sharing modes for CWNs. However, the performances of these two modes will be significantly changed in different circumstances. For example, when the PUs' traffic is low, which means the portion of spectrum occupied by PUs is small or the occupation time is short, the performance of overlay will better than that of underlay, therefore, CWNs should choose the overlay mode. However, on the other hand, when the PUs' traffic is heavy, which means the portion of spectrum occupied by PUs is large or the occupation time is long, SUs should choose the underlay mode because unoccupied spectrum is hard to find. Therefore, DSA protocols need to dynamically change the spectrum sharing mode to obtain the optimal network performance.

\subsection{Protocols with cooperative spectrum sensing based on cross-layer design}

Most existing distributed DSA protocols are designed based on the local spectrum sensing. However, local 
spectrum sensing usually cannot guarantee the accuracy of the sensing results due to the noise uncertainty, shadowing, and channel fading. Cooperative spectrum sensing is an efficient method to solve above problem. Although cooperative spectrum sensing and DSA protocols have been extensively studied in the literature, they are usually designed separately. The cooperative spectrum sensing is responsible for improve the accuracy of sensing results while the DSA protocols mainly aim to increase the network performances based on the determined spectrum opportunities. However, the two aspects are often unavoidable coupled with each other and should be jointly designed. For example, the spectrum opportunities vary with the transmit power. Then, the number of nodes involved in spectrum sensing and the energy-detection threshold will change accordingly. Therefore, in order to increase the accuracy of sensing results and further improve the network performances, the cooperative spectrum sensing and distributed DSA protocols should be jointly designed based on the crosslayer approach.

\subsection{Distributed collision avoidance mechanism}

In traditional multi-channel wireless networks, the collision avoidance mechanism is used to reduce the collisions among different users through spectrum access scheduling, channel allocation, and power control. However, due to the dynamically available spectrum resources, additional interference power constraints, and the absence of central controller, the collision avoidance mechanism is more complex in distributed CWNs. On the one hand, not only the collisions among SUs need to be considered, but also collisions between SUs and PUs should be taken into consideration. On the other hand, dynamically varying spectrum resources is a challenge for efficient spectrum access scheduling and channel allocation. Moreover, to guarantee that the total interference power caused by multiple SUs do not exceed the interference threshold through completely distributed manner is difficult to implement. Therefore, the distributed collision avoidance mechanism is worth for further studying.

\section{Conclusions}

In this article, we first addressed the challenges in the design and implementation of distributed dynamic spectrum access protocols. Then, we categorized the existing dynamic spectrum access protocols based on different criteria, such as spectrum sharing modes, spectrum allocation behaviors, spectrum access modes, the usage common control channel, spectrum usage strategies, the number of radios, and spectrum sensing techniques. We also discussed the advantages and disadvantages of each category under diverse classification criterion. Moreover, we made a comprehensive survey of the state-of-the-art distributed dynamic spectrum access protocols. In addition, we also reviewed the ongoing standardization efforts. Finally, we presented several open research issues for the dynamic spectrum access protocols, including spectrum handoff based protocols, spectrum prediction based protocols, adaptation of the spectrumsharing modes, protocols with cooperative spectrum sensing, and collision avoidance mechanisms.

\section{Endnote}

${ }^{\mathrm{a}}$ The spectrum assess protocols are typically implemented in the medium access control (MAC) layer, and therefore, we use the terms "spectrum access protocols" and "MAC protocols" exchangeably in the rest of this article.

\section{Acknowledgements}

The research reported in this article was supported in part by the National Natural Science Foundation of China under Grant No. 61172091, the National Science and Technology Major Project under Grant No. 2010ZX03005-003, the Specialized Research Fund for the Doctoral Program of Higher Education under Grant No. 20110201120014, and the National HiTech Research and Development Program of China (863) under Grant No. 2011AA01A105.

\section{Authors' contributions}

PR conceived of the study, participated in its design and coordination. YW designed the study and drafted the manuscript. QD participated in the manuscript's design and provided valuable suggestions. JX read the manuscript and participated in its coordination. All authors read and approved the final manuscript.

\section{Competing interests}

The authors declare that they have no competing interests.

Received: 30 April 2011 Accepted: 24 February 2012

Published: 24 February 2012

\section{References}

1. IEEE 802.22 Working Group on Wireless Regional Area Networks, http:// Www.ieee802.org/22/

2. CR Stevenson, J Chouinard, Z Lei, W Hu, SJ Shellhammer, W Caldwell, IEEE 802.22: The first cognitive radio wireless regional area network standard. IEEE Commun Mag. 47(1), 130-138 (2009)

3. J Wang, MS Song, S Santhiveeran, K Lim, G Ko, K Kim, SH Hwang, M Ghosh, $\checkmark$ Gaddam, K Challapali, First cognitive radio networking standard for personal/portable devices in TV white spaces, in IEEE DYSPAN, Singapore, pp. 1-12 (2010)

4. Standard ECMA-392, MAC and PHY for operation in TV white space. http:// www.ecmainternational.org/publications/standards/Ecma-392.htm (Dec. 2009)

5. R Kennedy, P Ecclesine, IEEE P802.11 af Tutorial. (2010) IEEE 802.11-10/ $0742 \mathrm{rO}$

6. J Mitola, GQ Maguire, Cognitive radio: making software radios more personal. IEEE Personal Commun. 6(4), 13-18 (1999). doi:10.1109/98.788210

7. ACV Gummalla, JO Limb, Wireless medium access control protocols. IEEE Commun Surv Tutorials. 3(2), 2-15 (2000)

8. S Kumar, VS Raghavan, J Deng, Medium access control protocols for ad hoc wireless networks. Ad Hoc Netw. 4(3), 326-358 (2006). doi:10.1016/j. adhoc.2004.10.001

9. J Mo, HW So, J Walrand, Comparison of multichanel MAC protocols. IEEE Trans Mobile Comput. 7(1), 50-65 (2008)

10. Q Zhao, BM Sadler, A survey of dynamic spectrum access: signal processing, networking, and regulatory policy. IEEE Signal Process Mag. 24(3), 79-89 (2007) 
11. C Cormio, KR Chowdhury, A survey on MAC protocols for cognitive radio networks. Ad Hoc Netw. 7(7), 1315-1329 (2009). doi:10.1016/j. adhoc.2009.01.002

12. TV Krishna, A Das, A survey on MAC protocols in OSA networks. Comput Netw. 53(9), 1377-1394 (2009). doi:10.1016/j.comnet.2009.01.003

13. HAB Salameh, M Krunz, Channel access protocols for multihop opportunistic networks: challenges and recent developments. IEEE Netw. 23(4), 14-19 (2009)

14. IF Akyildiz, WY Lee, KRL Chowdhury, CRAHNs: cognitive radio ad hoc networks. Ad Hoc Netw. 7(5), 810-836 (2009). doi:10.1016/j. adhoc.2009.01.001

15. J Xiang, Y Zhang, T Skeie, Medium access control protocols in cognitive radio networks. Wirel Commun Mobile Comput. 10(1), 31-49 (2010). doi:10.1002/wcm.906

16. F Khozeimeh, S Haykin, Dynamic spectrum management for cognitive radio: an overview. Wirel Commun Mobile Comput. 9(11), 1447-1459 (2009). doi:10.1002/wcm.732

17. G Bianchi, Performance analysis of the IEEE 802.11 distributed coordination function. IEEE J Sel Areas Commun. 18(3), 535-547 (2000). doi:10.1109/ 49.840210

18. J So, N Vaidya, Multi-channel MAC for ad hoc networks: handing multichannel hidden terminal using a signle transceiver, in ACM Inter Symposium on Mobile Ad Hoc Networking and Comupt, Tokyo, Japan, pp. 222-233 (2004)

19. P Pawelcazk, S Pollin, HS Wilson, ARS Bahai, RV Prasad, R Hekmat, Performance analysis fo multichannel medium access control algorithms for opportunistic spectrum access. IEEE Trans Veh Technol. 58(6), 3014-3031 (2009)

20. SL Wu, CY Lin, YC Tseng, JP Sheu, A new multi-channel MAC protocol with on-demand channel assignment for multi-hop mobile ad hoc networks, in IEEE DYSPAN, Maryland, USA, pp. 203-213 (2005)

21. SJ Yoo, H Nan, TI Hyon, DCR-MAC: distributed cognitive radio MAC protocol for wireless ad hoc networks. Wirel Commun Mobile Comput. 9(5), 631-653 (2009). doi:10.1002/wcm.610

22. H Su, X Zhang, Cross-layer based opportunistic MAC protocols for QoS provisionings over cognitive radio wireless networks. IEEE J Sel Areas Commun. 26(1), 118-129 (2008)

23. M Timmers, S Pollin, A Dejonghe, A distributed multichannel MAC protocol for multihop cognitive radio networks. IEEE Trans Veh Technol. 59(1), 446-459 (2010)

24. J Jia, Q Zhang, X Shen, HC-MAC: A Hardware-Constrained Cognitive MAC for Efficient Spectrum Management. IEEE J Sel Areas Commun. 26(1), 106-117 (2008)

25. C Cordeiro, K Challapali, C-MAC: a cognitive MAC protocol for multi-channel wireless networks, in IEEE DySPAN, Dublin, Ireland, pp. 147-157 (2007)

26. YR Kondareddy, P Agrawal, Synchronized MAC protocol for multi-hop cognitive radio networks, in ICC, Beijing, China, pp. 3198-3302 (2008)

27. CF Shih, TY Wu, W Liao, DH-MAC: a dynamic channel hopping MAC protocol for cognitive radio networks, in ICC, Cape Town, South Africa, pp. $1-5(2010)$

28. WY Lee, IF Akyildiz, Optimal spectrum sensing framework for cognitive radio networks. IEEE Trans Wirel Commun. 7(10), 3845-3857 (2008)

29. FF Digham, MS Alouini, MK Simon, On the energy detection of unknown signals over fading channels. IEEE Trans Commun. 55(1), 21-24 (2007)

30. $Y$ Zeng, $Y$ Liang, Spectrum-sensing algorithms for cognitive radio based on statistical covariances. IEEE Trans Veh Technol. 58(4), 1804-1815 (2009)

31. Y Zeng, Y Liang, Eigenvalue-based spectrum sensing algorithms for cognitive radio. IEEE Trans Commun. 57(6), 1784-1793 (2009)

32. R Zhang, T Lim, Y Liang, Y Zeng, Multi-antenna based spectrum sensing for cognitive radios: a GLRT approach. IEEE Trans Commun. 58(1), $84-88$ (2010)

33. Y Liang, Y Zeng, ECY Peh, AT Hoang, Sensing-throughput tradeoff for cognitive radio networks. IEEE Trans Wirel Commun. 7(4), 1326-1337 (2008)

34. Y Chen, Q Zhao, A Swami, Joint design and separation principle for opportunistic spectrum access in the presence of sensing errors. IEEE Trans Inf Theory. 54(5), 2053-2071 (2008)

35. Q Zhao, L Tong, A Swami, Y Chen, Decentralized cognitive MAC for opportunistic spectrum access in ad hoc networks: a POMDP framework. IEEE J Sel Areas Commun. 25(3), 589-600 (2007)

36. Q Zhao, B Krishnamachari, K Liu, On myopic sensing for multi-channel opportunistic access: structure, optimality, and performance. IEEE Trans Wirel Commun. 7(12), 5431-5440 (2008)
37. Y Chen, Q Zhao, A Swami, Distributed spectrum sensing and access in cognitive radio networks with energy constraint. IEEE Trans Signal Process. 57(2), 783-797 (2009)

38. AT Hoang, YC Liang, DTC Wong, YH Zeng, R Zhang, Opportunistic spectrum access for energy-constrained cognitive radios. IEEE Trans Wirel Commun. 8(3), 1206-1211 (2009)

39. Y Wang, P Ren, Z Su, A POMDP based Distributed Adaptive Opportunistic Spectrum. IElCE Trans Commun. E94-B(6), 1621-1624 (2011). doi:10.1587/ transcom.E94.B.1621

40. Y Wang, P Ren, G Wu, A throughput-aimed MAC protocol with QoS provision for cognitive ad hoc networks. IEICE Trans Commun. E93-B(6), 1426-1429 (2010). doi:10.1587/transcom.E93.B.1426

41. F Wang, M Krunz, S Cui, Price-based spectrum management in cognitive radio networks. IEEE J Selec Topics Signal process. 2(1), 74-87 (2008)

42. Y Wang, P Ren, Z Su, Polarization based Long-range Communication Directional MAC Protocol for Cognitive Ad Hoc Networks. IEICE Trans Commun. E94-B(5), 1265-1275 (2011). doi:10.1587/transcom.E94.B.1265

43. HAB Salameh, MM Krunz, O Younis, MAC protocol for opportunistic cognitive radio networks with soft guarantees. IEEE Trans Mobile Comput. 8(10), 1339-1352 (2009)

44. HAB Salameh, MM Krunz, O Younis, Cooperative adaptive spectrum sharing in cognitive radio networks. IEEE/ACM Trans Netw. 18(4), 1181-1194 (2010)

45. W Ren, Q Zhao, A Swami, Power control in cognitive radio networks: how to cross a multi-lane highway. IEEE J Sel Areas Commun. 27(7), 1283-1296 (2009)

46. X Wang, A Wong, PH Ho, Stochastic Medium Access for Cognitive Radio Ad Hoc Networks. IEEE J Sel Areas Commun. 29(4), 770-783 (2011)

47. B Hamdaouli, KG Shin, OS-MAC: an efficient MAC protocol for spectrumagile wireless networks. IEEE Trans Mobile Comput. 7(8), 915-930 (2008)

48. R Ramanathan, J Redi, C Santivanez, D Wiggins, S Polit, Ad hoc networking with direction antennas: a complete system solution. IEEE J Sel Areas Commun. 23(3), 496-506 (2005)

49. R Choudhury, $X$ Yang, R Ramanathan, NH Vaidya, On designing MAC protocols for wireless networks using directional antennas. IEEE Trans Mobile Comput. 5(5), 992-998 (2006)

50. X Zhang, H Su, CREAM-MAC: cognitive radio-enabled multi-channel MAC protocol over dynamic spectrum access networks. IEEE J Sel Topics Signal process. 5(1), 110-123 (2011)

51. S Haykin, Cognitive radio: brain-empowered wireless communications. IEEE J Sel Areas Commun. 23(2), 201-220 (2005)

52. GN Iyer, YC Lim, Efficient multi-channel MAC protocol and channel allocation schemes for TDMA based cognitive radio networks, in ICCSP, Kerala, India, pp. 394-398 (2011)

53. L Ma, X Han, C-C Shen, Dynamic open spectrum sharing for wireless ad hoc networks, in IEEE DySPAN, Maryland, USA, pp. 203-213 (2005)

54. RR Chen, KH Teo, BF Boroujeny, Random access protocols for collaborative spectrum sensing in multi-band cognitive radio networks. IEEE I Sel Topics Signal process. 5(1), 124-1136 (2011)

55. S Yin, D Chen, Q Zhang, S Li, Prediction-based throughput optimization for dynamic spectrum access. IEEE Trans Veh Technol. 60(3), 1284-1289 (2011)

56. L Ma, C-C Shen, B Ryu, Signal-radio adaptive channel algorithm for spectrum agile wireless ad hoc networks, in IEEE DySPAN, Dublin, Ireland, pp. 547-558 (2007)

57. F Wang, M Krunz, S Cui, Price-Based Spectrum Management in Cognitive Radio Networks. IEEE J Sel Topics Signal process. 2(1), 74-87 (2008)

58. SHA Ahmad, MY Liu, T Javidi, Q Zhao, B Krishnamachari, Optimality of myopic sensing in multi-channel opportunistic access. IEEE Trans Inf Theory. 55(9), 4040-4050 (2009)

doi:10.1186/1687-1499-2012-60

Cite this article as: Ren et al:: A survey on dynamic spectrum access

protocols for distributed cognitive wireless networks. EURASIP Journal on Wireless Communications and Networking 2012 2012:60. 